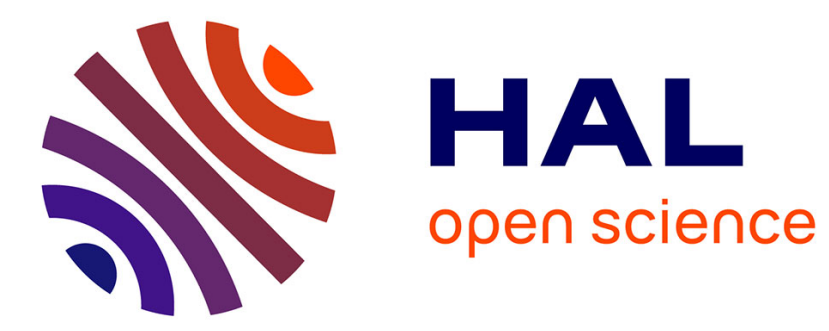

\title{
The optical depth sensor (ODS) for column dust opacity measurements and cloud detection on martian atmosphere
}

D. Toledo, Pascal Rannou, Jean-Pierre Pommereau, Thomas Foujols

\section{- To cite this version:}

D. Toledo, Pascal Rannou, Jean-Pierre Pommereau, Thomas Foujols. The optical depth sensor (ODS) for column dust opacity measurements and cloud detection on martian atmosphere. Experimental Astronomy, 2016, 42 (1), pp.61-83. 10.1007/s10686-016-9500-7 . insu-01322888

\section{HAL Id: insu-01322888 \\ https://hal-insu.archives-ouvertes.fr/insu-01322888}

Submitted on 4 Mar 2021

HAL is a multi-disciplinary open access archive for the deposit and dissemination of scientific research documents, whether they are published or not. The documents may come from teaching and research institutions in France or abroad, or from public or private research centers.
L'archive ouverte pluridisciplinaire HAL, est destinée au dépôt et à la diffusion de documents scientifiques de niveau recherche, publiés ou non, émanant des établissements d'enseignement et de recherche français ou étrangers, des laboratoires publics ou privés. 


\title{
The optical depth sensor (ODS) for column dust opacity measurements and cloud detection on martian atmosphere
}

\author{
D. Toledo ${ }^{1}$, P. Rannou ${ }^{1}$, J-P. Pommereau ${ }^{2}$ and T. Foujols ${ }^{2}$ \\ [1]\{GSMA, UMR 7331, CNRS, Université de Reims Champagne-Ardenne, Reims, \\ 51687, France $\}$ \\ [2]\{LATMOS, Université de Versailles-St-Quentin, GUYANCOURT, France\} \\ Correspondence to: D. Toledo (dani_toled@hotmail.com)
}

\begin{abstract}
A lightweight and sophisticated optical depth sensor (ODS) able to measure alternatively scattered flux at zenith and the sum of the direct flux and the scattered flux in blue and red has been developed to work in martian environment. The principal goals of ODS are to perform measurements of the daily mean dust opacity and to retrieve the altitude and optical depth of high altitude clouds at twilight, crucial parameters in the understanding of martian meteorology.

The retrieval procedure of dust opacity is based on the use of radiative transfer simulations reproducing observed changes in the solar flux during the day as a function of 4 free parameters: dust opacity in blue and red, and effective radius and effective width of dust size distribution. The detection of clouds is undertaken by looking at the time variation of the color index (CI), defined as the ratio between red and blue ODS channels, at twilight. The retrieval of altitude and optical depth of clouds is carried out using a radiative transfer model in spherical geometry to simulate the CI time variation at twilight. Here the different retrieval procedures to analyze ODS signals, as well as the results obtained in different sensitivity analysis are presented and discussed.
\end{abstract}

\section{Introduction}

On Mars, dust and clouds are primary elements for studying the evolution and behavior of the atmosphere (Gierasch and Goody, 1972). Ice clouds and dust particles interact with solar radiation, influencing directly the heating rates of the surface and atmosphere, and hence on the martian atmospheric circulation (e.g. Madeleine et al., 2011; Medvedev et al., 2011). In addition, in the absence of massive condensed water and precipitation, dust lifted from the surface of Mars by winds and atmospheric instability, are the unique condensation nuclei available at the atmosphere. These facts highlight the importance of dust and cloud particles in the vertical structure of the Mars atmosphere. Several years of earth-based, orbital and land observations have been analyzed to characterize the optical and physical properties of dust and clouds (e.g. Korablev et al., 1993; Pollack et al., 1995; Tomasko et al., 1999; Markiewicz et al., 1999; Montmessin et al., 2006; Wolff et al., 2006; Lemmon et al., 2015). Much progress has been made in simulating the meridional and vertical evolutions of atmospheric circulation. However, because dust and clouds are highly inhomogeneous and variable, more detailed information of the optical and physical properties are necessary. Orbital observations have provided information and constrains on optical properties of dust and clouds (Montmessin et al., 2006; Smith, 2009; Wolff et al., 2006). Such properties are for instance droplet size, dust size distribution, dust optical depth.

While orbital observations provide a more complete global coverage and observations, the available accuracy of aerosol characterization is often not enough 
(e.g., Smith, 2004; Wolff et al., 2009; Lemmon et al., 2015). Despite that Lander observations do not provide global coverage, their wide angular measurements of sky brightness are best suited to retrieve detailed information at key locations. The first estimations of dust opacity from surface observations were carried out using Viking and Pathfinder measurements (e.g. Pollack et al., 1979; Colburn et al., 1989; Smith and Lemmon, 1999). In addition, Pollack et al. (1995), Tomasko et al. (1999) and Markiewicz et al. (1999) derived different values of geometric cross section weighted mean radius $r_{\text {eff }}$ and variance $v_{\text {eff }}$ of the dust size distribution by comparing the sky brightness observations taken from Viking Lander 1 and 2, and Pathfinder with radiative transfer simulations. These authors provided information on phase function and single scattering albedo of dust particles using a semi empirical method (Pollack and Cuzzi, 1980) designed to account for non-sphericity of particles. Subsequently, the rovers from Mars Exploration Rover mission have provided measurements of dust optical depth over a total of 5 Mars years (Lemmon et al., 2004, 2015), revealing a strong year to year variability in dust load.

In this work, we present the lightweight optical depth sensor (ODS) instrument, designed to measure the dust opacity from the surface of Mars, as well as to characterize clouds at twilight. The first ODS prototype was developed for the failed MARS96 ROSKOSMOS/CNES mission whose principal objective was to built an instrument as simple, energy saving and lightweight as possible for operating during several martian years. It was subsequently proposed as a component of the NASA PASCAL Mars meteorological network, mission which did not reach the operational phase partly owing to its high technical risk. It was also proposed for the CNES NETLANDER mission, unfortunately cancelled in 2003. The version of ODS proposed for these missions included improvements in the optics of the instrument with respect to the MARS96 version. ODS was selected through an ESA/NASA AO in the payload of the atmospheric package DREAMS onboard the MARS 2016 mission (not included anymore in the payload because of funding issues). Additional developments for this mission resulted in a mass reduction and a technical simplification of the ODS optical head. Finally, ODS is included in the METEO meteorological station on board the ExoMars 2018 Lander, thanks to the support of the Russian Space Science Institute (IKI).

The goal of this paper is to describe the main concept of the instrument as well as the retrieval procedures adopted to obtain as much information as possible on the optical properties of martian aerosol from ODS signals.

\section{Instrumentation}

\subsection{Principle of measurements}

By using a single sensor for each wavelength range, ODS is designed to make alternative observations of the scattered flux at zenith and the sum of direct flux and scattered flux during the course of the day. Figure 1 shows the simulated scattered and direct flux on Mars surface as function of dust opacity. The ratio of direct flux to scattered flux is a monotonic function of dust opacity, providing an estimation of the dust opacity independent of the sensor calibration.

The detection of high altitude clouds in the martian atmosphere is carried out by looking at the relative evolution of scattered flux in blue and red at twilight. The ratio between the scattered flux in red and blue, defined as the color index (CI), varies with solar zenith angle (SZA) and when high altitude clouds are present, they produce a 
minimum in the time variation of CI during sunrise or sunset, whose SZA of minimum CI increases with cloud altitude. As discussed later, multiple scattering resulting from the forward scattering peak of dust particles makes a major contribution to the intensity of the twilight, and due to the fact that the forward peak of phase function of martian dust is larger in blue than in red (see Figure 2) the atmosphere takes on a bluish glow. This method for deriving cloud altitude from CI observations is similar to that developed for Polar Stratospheric Clouds (Sarkissian et al., 1991).

\subsection{ODS instrument}

The Martian prototype of ODS is a lightweight $(107 \mathrm{~g})$ optical instrument looking at zenith (Maria et al., 2006; Tran et al., 2005), and made of two identical optical heads. Each head includes a 1-mm aperture, which permits entry of scattered and direct solar light on to an upward-oriented primary parabolic mirror (left of Figure 3). The light from the primary mirror is directed to a downward oriented secondary parabolic mirror, which then directs the flux to a photodiode. Both parabolic mirrors are surrounded by vertical black walls (preventing the reflection of the light) that together with a 2-mm central mask located below the 1-mm aperture define an annular field of view (FOV) shown on the right of Figure 3. The 2-mm central mask also would serve as a dust collection platform, to minimize dust accumulation on the primary mirror surface. Blue and red channels are selected by using coloured glass filters placed in front of the photodiodes. The only differences between the two ODS heads are the filters in front of the detectors. The FOV would permit for each ODS head alternative measurements of the scattered flux at zenith and the sum of direct flux and scattered flux. Finally the photodiode current is amplified by a 8-decade logarithmic amplifier, allowing measurements over a large range of dust opacities.

\section{Dust optical depth measurement}

The daily average optical depth of martian dust is estimated by simulating ODS signals throughout the day with a radiative transfer model. The photodiode current is calculated by:

$$
\begin{aligned}
& i=S_{\text {pupil }} \int_{0}^{\infty} T_{\text {filter }}(\lambda) R_{\text {diode }}(\lambda) T_{\text {mask }}\left(\mu_{0}, \phi_{0}, \lambda\right) \mu_{0} \Phi \exp \left(-\tau / \mu_{0}\right) d \lambda+ \\
& S_{\text {pupil }} \int_{0}^{\infty} \int_{0}^{2 \pi} \int_{-1}^{0} I\left(\tau, \mu_{0}, \mu, \phi, \lambda\right) T_{\text {filter }}(\lambda) R_{\text {diode }}(\lambda) T_{\text {mask }}(\mu, \phi, \lambda) \mu d \mu d \phi d \lambda
\end{aligned}
$$

where, $I$ is the scattered intensity calculated by the radiative transfer model; $\mu_{0}, \mu$ and $\phi$ are the cosine of SZA, the cosine of observation zenith angle and the azimuth angle, respectively; $T_{\text {mask }}, T_{\text {filter, }} R_{\text {diode }}$ (intensity per power) and $\Phi$ are the mask transmission, the filter transmission, the spectral response of photodiode and the solar flux, respectively; $\tau$ the total vertical opacity, $S_{\text {pupil }}$ the area of the pupil and $\lambda$ the wavelength. First term of right side of Equation (1) is the contribution from direct light. The scattered intensity field I for different values of dust opacity can be easily calculated through a radiative transfer model. The final conversion into output voltage is done using the electronic output function.

Our goal here is to calculate the scattered intensity field as seen from surface of Mars as function of dust opacity and atmospheric properties. Modelling the scattered 
intensity field requires the following dust optical properties: optical depth $\tau$; phase function $\mathrm{P}(\cos \Theta)$, and single scattering albedo $\omega$. Therefore, a method to calculate the phase function and single scattering albedo for non-spherical particles is required as well as a radiative transfer code to compute the scattered intensity field. Since dust optical depth retrievals are carried out during daytime, the scattered intensity field is computed by using the DISORT (Discrete Ordinates Radiative Transfer Program for a Multi-Layered Plane-Parallel Medium) code (Stamnes et al., 1988), which is a 1dimensional radiative transfer model in plane parallel geometry. In order to test the plane parallel approximation, DISORT computations were compared against a tested Monte Carlo radiative transfer code in spherical geometry (Trân, 2005) for SZAs smaller than $90^{\circ}$. The results showed the reliability of DISORT simulations at SZAs between 0 and $80^{\circ}$ (Toledo, 2015).

\subsection{Optical properties of martian dust}

Since our goal is to test the sensitivity of ODS to the optical properties of dust particles, for practical reasons the phase function and single scattering albedo are derived from the semi empirical method proposed by Pollack and Cuzzi, (1980). However, we emphasize here that once defined the different parameters in the retrieval procedure based on the sensitivity tests of ODS signal, the scattering properties of dust particles will be approximated using the T-matrix method (Mishchenko et al., 1997) for the analysis of a real ODS signal on Mars.

This semi empirical method approximates the optical properties of dust particles by using a total of four parameters $X_{\text {large }}$, SAR, $\theta_{\min }$, SLP. The parameters $\theta_{\text {min }}$ and SLP are used to set up the phase function, $\theta_{\min }$ establishes the scattering angle where the phase function reaches its minimum, and SLP which defines the slope of the phase function at $0^{\circ}\left(S L P=-d \log P(\Theta) / d \Theta \mathrm{I}_{\Theta=0}\right)$. The parameter $\mathrm{X}_{\text {large }}$ establishes a critical size for which the scattering of particles is calculated by Mie theory or is approximated by geometrical optics. SAR is the ratio of irregular particle surface area to that of an equal spherical particle and it is used to weight the relative contribution by particles smaller than $X_{\text {large }}$ and greater than $X_{\text {large }}$ to the scattering properties. Based on results of different sensitivity analysis, Pollack et al. (1995) adopted a fixed values for $\mathrm{X}_{\text {large }}=5$ and $\mathrm{SAR}=1.3$ for martian dust particles. Following these authors as well as other works (e.g., Tomasko et al., 1999; Montmessin et al., 2002) we fixed the value of these parameters for the simulations. In addition to these parameters, the refractive index and size distribution of dust particles are needed. Different functions to describe the size distribution have been used. For instance, Tomasko et al. (1999) and Markiewicz et al. (1999) employed the gamma distribution given by Hansen and Travis, (1974). This function is expressed in the form

$$
n(r)=c r^{\left(1-3 v_{e f f}\right) / v_{e f f}} \exp \left(-r /\left(r_{e f f} v_{e f f}\right)\right)
$$

where $r, r_{\text {eff }}, v_{\text {eff }}$ are the particle radius, effective radius and effective width, respectively; $\mathrm{c}$ is a constant. However, other studies have chosen the lognormal size distribution (Montmessin, 2002; Pollack et al., 1995). In this work we take the gamma distribution as expressed in Equation (2). The election of the function to describe the size distribution is not crucial in the calculations since as was demonstrated by Hansen and Travis (1974), different forms of size distribution provide the same scattering properties as long as the first two moments ( $\mathrm{r}_{\text {eff }}$ and $\left.\mathrm{v}_{\mathrm{eff}}\right)$ are equal. The particle imaginary refractive index at blue and red wavelengths are taken from 
Tomasko et al. (1999), while the real part is fixed to 1.5 for both wavelengths (Pollack et al., 1995; Tomasko et al., 1999).

\subsection{Sensitivity to dust optical depth}

In order to test the sensitivity of ODS to dust opacity we have simulated ODS measurements on Mars surface for different dust loads. For the calculations of the solar trajectory, we assume that ODS is localized at $30^{\circ} \mathrm{N}$ latitude, $0^{\circ} \mathrm{W}$ longitude. It is assumed that the absorption and scattering coefficients decrease exponentially with altitude and with a scale height of $10 \mathrm{~km}$. However, the vertical distribution of dust does not have a major impact on the retrievals, since the radiative transfer simulations are carried out in plane-parallel geometry. Indeed, we tested different values of scale height $(8,9,10,11$ and $12 \mathrm{~km})$ as well as combinations of those scale heights with dust layers located at different altitudes, and in all cases we found similar results. Figure 4 shows the evolution of simulated ODS signals during the course of a martian day for different dust opacities and constant values of $r_{\text {eff }}, v_{\text {eff }}, \theta_{\min }$ and SLP ( $r_{\text {eff }}=1.6$ $\mu \mathrm{m}, v_{\text {eff }}=0.2, \quad \mathrm{SLP}_{\text {blue }}=115, \mathrm{SLP}_{\text {red }}=120, \theta_{\min }^{\text {blue }}=138^{\circ}, \theta_{\min }^{\text {blue }}=150^{\circ}$ ). The bands delimited by the black dashed lines indicate the time intervals for which the sun is within the FOV of ODS. The ODS signal along the day can be divided into two time intervals according to the signal variations: the first (09:00 to 11:00 UTC and 12:00 to 15:00 UTC) is characterized by a rapid increase and subsequently a rapid decrease of ODS signals as consequence of the direct solar flux and, the second (09:00 to 11:00 UTC and 12:00 to 15:00 UTC) corresponds to a lower variation of ODS signals with the time respect to the previous time intervals since only scattered flux is received by the instrument. By comparing ODS signal in both time intervals it is obvious that the ratio between the flux scattered by the atmosphere at zenith and the sum of the scattered flux and direct solar flux depends significantly on the dust load. Therefore, we can estimate the dust opacity by searching the optimal value of this parameter that provides the observed ODS signal.

\subsection{Sensitivity of ODS signal to other parameters}

In previous section we have shown the sensitivity of ODS signal to dust opacity. However, those simulations were carried on holding constant the others of parameters of our model: $r_{\text {eff }}, v_{\text {eff }}, \theta_{\min }$ and SLP. Since the scattering properties of dust particles depend on these parameters, a sensitivity analysis of ODS signals to size distribution as well as to the parameters to tune the phase function is necessary. We have chosen to begin this analysis by using the gamma distribution function for different $r_{\text {eff }}$ values and holding constant the other parameters. In order to simplify the simulations we have chosen the same total opacity in both wavelength ranges. However, note that the dust opacity measurements in each channel will be different due to the spectral dependence of this parameter. Figure 5 illustrates the ODS signals simulated for different $r_{\text {eff }}$ values and a total opacity of 0.4. Simulations indicate that $r_{\text {eff }}$ has an impact on the blue ODS signal, since some differences are found between the simulated blue signals for those values of $r_{\text {eff }}$ (left panel of Figure 5). However, those differences are not observed on the red channel (right panel of Figure 5). The values of the single scattering albedo and asymmetry parameter $(\mathrm{g})$ of the phase function obtained for those $r_{\text {eff }}$ values are shown in Table 1 . Indeed, we obtain a relative difference in the single scattering albedo for $r_{\text {eff }}=1.2 \mu \mathrm{m}$ and $r_{\text {eff }}=1.8 \mu \mathrm{m}$ of $1.0 \%$ in the red wavelength, whereas in the blue wavelength of $6.3 \%$. Same conclusions are derived for the phase functions by comparing the values of $g$ provided in Table 1 . In order to analyze the sensitivity of ODS signals to $r_{\text {eff }}$ and $\tau$ simultaneously, we have 
performed a contour analysis of these parameters. Since we cannot perform a contour analysis with a real ODS signal, we simulate first ODS signals for specific values of $r_{\text {eff }}$ and $\tau$, and subsequently we add a random noise of an amplitude of $5 \%$. These signals are supposed to be the ODS measurements on Mars $\left(\mathrm{O}_{\text {Mars }}\right)$. According to this formulation the chi-square is defined as follows:

$\chi^{2}\left(\tau, r_{\text {eff }}\right)=\frac{1}{n} \sum_{i=1}^{n}\left(O_{\text {Mars }}(i)-O_{\text {simul }}\left(i, \tau, r_{\text {eff }}\right)\right)^{2}$

where $\mathrm{O}_{\text {simul }}$ is the simulated ODS signal in the $\tau$-r $\mathrm{r}_{\text {eff }}$ space and $\mathrm{n}$ is the number of observations. Figure 6 illustrates contours of $\chi^{2}$, where $\mathrm{O}_{\text {Mars }}$ was computed for $\tau=0.4$ and $\mathrm{r}_{\mathrm{eff}}=1.4 \mu \mathrm{m}$, and where each $\mathrm{k}$ contour line has a constant value of $\chi^{2}=2^{k} \bullet \chi_{\text {min }}^{2}(k=1,2 \ldots)$. Therefore, the value of $\chi^{2}$ for the dash-dot lines in Figure 6 is $2 \cdot \chi_{\text {min }}^{2}$. We can observe that the red channel is practically insensitive to $r_{\text {eff }}$ for that range of values, since the different contours lines of $\chi^{2}$ are almost verticals. However, Figure 6 demonstrates that $r_{\text {eff }}$ has an impact on the blue ODS signal, where $\mathrm{r}_{\text {eff }}$ takes values between $1.22 \mu \mathrm{m}$ and $1.59 \mu \mathrm{m}$ for $\chi^{2}=2 \cdot \chi^{2}{ }_{\text {min }}$, and $\tau$ between 0.35 and 0.46. A similar sensitivity analysis has been performed for $v_{\text {eff, }}$ whose results are shown in Figure 7 and in Table 2. While red ODS signal is not sensitive to $v_{\text {eff }}$ for that range of values, some differences are found in the simulated blue signals. Note that the relative difference in the single scattering albedo for $\nu_{\text {eff }}=0.2 \mu \mathrm{m}$ and $\nu_{\text {eff }}=0.6 \mu \mathrm{m}$ is only $4 \%$ in the blue wavelength range. Figure 8 illustrates contours of $\chi^{2}$ in the $\tau$ $\nu_{\text {eff }}$ space for a $\mathrm{O}_{\text {Mars }}$ signal computed for $\tau=0.4$ and $\nu_{\text {eff }}=0.6$. Note that the red channel is almost insensitive to $v_{\text {eff }}$ for that range of values. However, we observe that for the contour line corresponding to $\chi^{2}=2 \cdot \chi^{2}{ }_{\text {min }}, v_{\text {eff }}$ takes values between 0.44 and 0.87 , indicating that the blue ODS channel is sensitive to this parameter. In summary, results show that red ODS channel is slightly affected by $r_{\text {eff }}$ and $v_{\text {eff }}$ under moderate dusty conditions, whereas for blue channel these parameters should to be taken into account in the design of the retrieval algorithm.

A similar sensitivity analysis was performed for the parameters to tune the phase function, $\theta_{\min }$ and SLP. Note that only the phase function of dust particles is affected by variations in $\theta_{\min }$ and SLP. Hence this sensitivity analysis in turn will allow us to study if the changes observed in the ODS signals when varying $r_{\text {eff }}$ and $v_{\text {eff }}$ are mostly due to the changes in the single scattering albedo or in the phase function of dust particles. Different phase functions were computed, allowing SLP to vary between 80 and 140 and $\theta_{\min }$ between $110^{\circ}$ and $170^{\circ}$. Despite that the phase function depends on SLP and $\theta_{\min }$ (left panel of Figure 9), we find that ODS signals do not significantly suffer with variations in these two parameters. For instance, the right panel of Figure 9 shows the correlation between simulated ODS signals for $\theta_{\min }=170^{\circ}$ and $\mathrm{SLP}=115$, and $\theta_{\min }=110^{\circ}$ and SLP $=115$. We observe that the correlation between both signals is very close to the identity relation. This is due to the fact that the parameters to tune the phase function mainly affect the part of the phase function with scattering angles higher than 90 degrees (backscattering), and ODS is essentially sensitive to the forward scattering. These results indicate that the variations observed in the ODS signals by changing $r_{\text {eff }}$ and $v_{\text {eff }}$ are mainly due to variations in the phase function for small scattering angles, as well as to the variations in the single scattering albedo. This demonstrates the robustness of ODS measurements against errors in the phase 
function at scattering angles larger than $90^{\circ}$. That is to say, the part of the phase function that is more affected by the shape of the particles.

So far, the different sensitivity tests have been performed for low dust loading conditions. The different ranges of values were chosen to cover the possible values of these parameters according to previous studies. Thus, a similar analysis have been performed under dust storm conditions, where the optical depth may reach values up or above 2, with a wide range of values of $r_{\text {eff }}$ and $v_{\text {eff. }}$. Figure 10 shows the simulated ODS signals for values of $r_{\text {eff }}$ ranging from $1 \mu \mathrm{m}$ to $3 \mu \mathrm{m}$ and a total opacity of 2 . In contrast to the simulations under moderate dust load conditions, in this case the red channel is also affected by $r_{\text {eff. }}$. The single scattering albedo and asymmetry parameter of the phase function calculated for those $r_{\text {eff }}$ values are summarized in Table 3 . Noticeable differences in the single scattering albedo are found for those $r_{\text {eff }}$ values, a relative difference between $r_{\text {eff }}=1 \mu \mathrm{m}$ and $r_{\text {eff }}=3 \mu \mathrm{m}$ of $6.2 \%$ in the red wavelength range, whereas in the blue wavelength range of $14.6 \%$. Figure 11 illustrates contours of $\chi^{2}$ in the $\tau-r_{\text {eff }}$ space for a $\mathrm{O}_{\text {Mars }}$ signal computed for $\tau=2$ and $r_{\text {eff }}=1.6 \mu \mathrm{m}$. As the case under moderate dusty conditions, the red ODS channel is practically insensitive to $r_{\text {eff. }}$ Indeed, the contour line corresponding to $\chi^{2}=2 \cdot \chi_{\text {min }}^{2}$ goes from values of $r_{\text {eff }}$ near to $1.3 \mu \mathrm{m}$ to values higher than $2 \mu \mathrm{m}$. Regarding the blue channel, we see the same results as found under moderate dusty conditions. A similar sensitivity analysis has been performed for $v_{\text {eff }}$ under dust storm conditions, whose results are shown in Figure 12 and in Table 4. As under moderate dust load conditions, the red ODS channel is not affected by this parameter, whereas in the blue channel some differences are found in the simulations. Contour plots of $\chi^{2}$ in the $\tau-v_{\text {eff }}$ space are shown in Figure 13. We see that the contour lines in the blue channel case reveal the need to include $v_{\text {eff }}$ in the estimation of the opacity of dust, whereas the red channel is practically insensitive to this parameter. Finally, we tested the effect of changing the parameters to tune the phase function under dust storm conditions, where we find similar results as showed in Figure 9.

In summary, results demonstrate that ODS signals are mainly dominated by the opacity of dust. However, we observe in the simulations that the parameters to describe the size distribution ( $\mathrm{r}_{\text {eff }}$ and $\nu_{\text {eff }}$ ) should be considered as free parameters in the retrieval algorithm for dust opacity. The parameters controlling the shape of the phase function in backscattering can be fixed to constant values according to our results. In addition, all these test simulations were undertaken for different values of surface albedo, obtaining in all cases the same results.

\subsection{Dust opacity retrieval procedure}

Our goal here is to define a reliable retrieval procedure to retrieve the dust opacity, based on the results obtained in previous section. As was seen on Figure 1 and Figure 4 , the ratio between the scattered flux at zenith and and the sum of the direct flux and the scattered flux highly depends on the dust opacity. Therefore, given an ODS signal we can estimate the dust opacity by searching the optimal value of this parameter that provides the best fit between observations and simulations. An important point here is that the fit between observations and simulations is carried out for the shape of ODS signals, being therefore independent of any absolute calibration of the instrument. However, the parameters to describe the size distribution ( $r_{\text {eff }}$ and $v_{\text {eff }}$ ) should be included as free parameters in our retrieval procedure. By using both ODS signals, a total of four free parameters are considered in the retrieval procedure: the dust opacity in the blue and red wavelength ranges $\left(\tau_{\text {blue }}, \tau_{\text {red }}\right)$ and the effective radius and 
effective width ( $\left.\mathrm{r}_{\text {eff }}, v_{\text {eff }}\right)$ of the dust size distribution. We could have linked $\tau_{\text {blue }}$ and $\tau_{\text {red }}$ to $r_{\text {eff }}$ and $v_{\text {eff }}$ through a total number of particles, but fixing separately the opacities allows more flexibility for the algorithm. Note that from $\tau_{\text {red }}$ and $\tau_{\text {red }}$ retrievals, the Angström exponent is also provided by ODS. This retrieval procedure was tested against AERONET (Aerosol Robotic NETwork) retrievals in a test campaign in Ouagadougou, a Mars-like desert location in the sahelian region of Burkina-Faso in West Africa (Toledo et al., 2016). The retrieved daily average dust opacity of both instruments showed a correlation coefficient of 0.94 considering in the comparison only the cloud-free days (the relevant case for Mars). In addition, in this campaign was tested the possibility of providing two measurements of dust opacity per day (in the morning from 07:00 to 12:00 UTC and the afternoon from 12:00 to 16:30 UTC) for days of high dust opacity variability. In this second comparison, it was found a correlation coefficient between both instruments of 0.97 .

Since radiative transfer simulations take long calculation time, the retrieval procedure makes use of a pre-computed set of look-up tables (LUT), for minimizing the mean squares difference between simulated and observed ODS signals. In addition, an error is provided by the square of diagonal elements of parameters covariance matrix

$$
\sigma=\sqrt{\operatorname{diag}\left(\left[J^{T} W J\right]^{-1}\right)}
$$

where $\mathrm{J}$ is the Jacobian matrix and $\mathrm{W}$ a diagonal matrix with $W_{i i}=\frac{1}{w_{i}}$ entries, being $w_{i}$ the error associated to measurement $y_{i}$.

\subsection{ODS orientation}

In this part, we test the ability of ODS to retrieve the dust properties and the orientation. We use real data of the Ouagadougou campaign but with a sampling comparable to what is expected for a martian mission, and leaving the orientation as a free parameter. It is essential to know the tilt and orientation of ODS relative to the local axis before the ODS measurements can be compared with the simulations. In order to define a procedure able to retrieve these angles it is convenient to specify the orientation of ODS using a fixed local reference system $L\{x, y, z\}$ where $z$ is the zenith, and $\mathrm{x}$ and $\mathrm{y}$ are the East and North directions. To describe the ODS viewing directions with respect to the local reference system $\mathrm{L}$, we introduce a right-handed coordinate system $\mathrm{O}\left\{\mathrm{x}^{\prime}, \mathrm{y}^{\prime}, \mathrm{z}^{\prime}\right\}$ affixed to ODS and having the same origin as L. The direction $z$ ' is the ODS vertical axis, $x^{\prime}$ is the direction from the centre of the mask towards one of the mask legs and $y^{\prime}$ is perpendicular to $z$ ' and $x$ ' according to the right hand rule. The tilt and orientation of ODS respect to $L$ are specified by the Euler angles $(\alpha, \beta$ and $\gamma)$ which transform the local reference system L into the ODS coordinate system $\mathrm{O}$.

In order to define the Euler angles we first introduce an additional axis referred as line of nodes. This axis is defined as the intersection of the xy and the x'y' coordinate planes, and hence it is perpendicular to zz' plane. The three Euler angles are then defined as follows: 1) $\alpha$ is a rotation around the $z$ axis and is defined as the angle between the $\mathrm{x}$ axis and the line of nodes; $\beta$ is a rotation around the line of nodes and is defined as the angle between the $\mathrm{z}$ axis and the $\mathrm{z}$ ' axis; and $\gamma$ is a rotation around the $z$ ' axis and is defined as the angle between the line of nodes and the $x$ ' axis. Figure 14 shows the coordinate systems $\mathrm{L}\{\mathrm{x}, \mathrm{y}, \mathrm{z}\}$ and $\mathrm{O}\left\{\mathrm{x}^{\prime}, \mathrm{y}^{\prime}, \mathrm{z}^{\prime}\right\}$ for a given set of Euler 
angles. Note that $\beta$ represents the tilt of the instrument. The rotation matrix that transforms the fixed local reference system L into the ODS coordinate system $\mathrm{O}$ is given by:

$$
M=\left[\begin{array}{ccc}
\cos \alpha \cos \beta \cos \gamma-\sin \alpha \sin \gamma & \sin \alpha \cos \beta \cos \gamma+\cos \alpha \sin \gamma & -\sin \beta \cos \gamma \\
-\cos \alpha \cos \beta \sin \gamma-\sin \alpha \cos \gamma & -\sin \alpha \cos \beta \sin \gamma+\cos \alpha \cos \gamma & \sin \beta \sin \gamma \\
\cos \alpha \sin \beta & \sin \alpha \sin \beta & \cos \beta
\end{array}\right]
$$

Therefore, if $\mathrm{T}_{\mathrm{lab}}(\mu, \phi)$ represents the transmission of the mask as a function of the zenith angle and the azimuth angle (see Equation (1)) measured in the laboratory and the angles of rotation are known, by using the matrix $M$ we can calculate the transmission $\mathrm{T}_{\text {mask }}(\mu, \phi)$ for the ODS orientation. Thus $\alpha, \beta$ and $\gamma$ are the angles for each channel that must be retrieved from ODS measurements. Figure 15 shows different orientations of ODS as well as the ODS signals simulated for those orientations. We can observe that depending on the tilt and orientation of ODS the sun is within the FOV of ODS for different time intervals. These simulations remark the importance of retrieving the tilt and orientation of ODS.

For the calculation of the rotation angles, we first select a day of ODS measurements. We then simulate the ODS signals for different values of $\alpha, \beta$ and $\gamma: \alpha$ is allowed to vary between $0^{\circ}$ to $360^{\circ}$ in steps of $4^{\circ}, \beta$ between $0^{\circ}$ to $2^{\circ}$ in steps of $0.4^{\circ}$ and $\gamma$ from $0^{\circ}$ to $360^{\circ}$ in steps of $4^{\circ}$. These simulations are carried out keeping constant the rest of parameters $\left(\tau_{\text {blue }}, \tau_{\text {red, }}, r_{\text {eff }}\right.$ and $\left.v_{\text {eff }}\right)$. For these different configurations of rotations, we select the rotation ( $\alpha_{\text {blue }}, \beta_{\text {blue }}, \gamma_{\text {blue }}, \alpha_{\text {red }}, \beta_{\text {red }}, \gamma_{\text {red }}$ ) that provides the minimum value of mean squared difference between observations and simulations. Note that we select three angles for each ODS channel because the two optical heads are not perfectly aligned. Subsequently, the final rotations are obtained by doing a best fit of $\alpha_{\text {blue }}, \beta$ blue, $\gamma_{\text {blue, }} \alpha_{\text {red }}, \beta_{\text {red, }} \gamma_{\text {red }}, \tau_{\text {blue }}, \tau_{\text {red, }}, r_{\text {eff }}$ and $v_{\text {eff }}$, using as initial angles for the search those obtained in the previous step. This method to retrieve the orientation of ODS was applied to the data collected by the terrestrial prototype of ODS in the campaign in Ouagadougou. Figure 16 shows, as an example, the evolution of ODS signals (blue and red channels) measured on 23 December 2004 in Ouagadougou, where the black dashed lines represent the results of applying this method to retrieve the angles of rotation. The retrieved Euler angles for each ODS channel as well as the orientation $\Phi$, defined as the angle between $\mathrm{x}$-axis and the projection of $\mathrm{x}$ '-axis over $\mathrm{x}$-y plane, are illustrated in Table 5. In order to have a better estimation of the angles, the fit was carried out for the time interval for which the sun is within the FOV of ODS. Once the angles of rotation are retrieved, the retrieval procedure for dust opacity presented in previous section can be applied to ODS measurements using the whole daytime ODS signals.

\subsection{Sampling strategy}

Despite that results indicate that the method presented in previous section retrieves correctly the orientation of ODS, a major difference between the terrestrial and martian ODS measurements is the daily sampling frequency. During the campaign in Ouagadougou, ODS observations were taken at 10-second interval in both channels, while for ODS measurements on Mars the observations would be acquired at larger time intervals. In order to test the reliability of the method to retrieve the orientation of ODS for different sampling frequencies, we retrieved the orientation of ODS using 
the same signals shown in Figure 16 but using only the instantaneous observations that were taken at 5-minute interval, then 10-minute, 15-minute, 30-minute and finally at 60-minute interval. Thus, 5 sets of Euler angles are retrieved $\left(\mathrm{O}_{5 \mathrm{~min}}, \mathrm{O}_{10 \mathrm{~min}}, \mathrm{O}_{15 \mathrm{~min}}\right.$, $\mathrm{O}_{30 \mathrm{~min}}$ and $\left.\mathrm{O}_{60 \mathrm{~min}}\right)$ for each channel. Figure 17 illustrates the tilt $(\beta)$ and orientation angle $\Phi$ retrieved in section 3.5 using the original ODS signals (grey bars) and those retrieved using the different sampling frequencies (black dots). With the exception of the 60-min sampling frequency, results indicate the reliability of the procedure to retrieve the tilt and orientation angles for those different sampling frequencies, since the retrieved angles are consistent with the errors (grey bars). We performed for each channel a linear regression between the ODS signal simulated using the orientation derived from all observations (10-second interval) and those simulated using $\mathrm{O}_{5 \mathrm{~min}}$, $\mathrm{O}_{10 \min }, \mathrm{O}_{15 \min }, \mathrm{O}_{30 \min }$ and $\mathrm{O}_{60 \mathrm{~min}}$. In all cases, the correlation of each regression was above 0.95 , showing therefore the capability of the method to retrieve the orientation of ODS for those different sampling frequencies.

In addition, we have selected a total of 7 days of measurements during campaign in Ouagadougou to study the robustness of the retrieval procedure for dust opacity in terms on the different sampling frequencies. Firstly, we retrieved for these days the dust opacity using the original signals (observations taken at 10 -second interval). Subsequently, we retrieved the dust opacity for same days but using only the observations that were taken at 5-minute interval, 10-minute, 15-minute, 30-minute and 60-minute interval. Thus, for each day and channel, dust opacity is retrieved for a total of 6 different sampling frequencies, and where for each sampling frequency we have used the corresponding retrieved orientation $\left(\mathrm{O}_{5 \mathrm{~min}}, \mathrm{O}_{10 \mathrm{~min}}, \mathrm{O}_{15 \mathrm{~min}}, \mathrm{O}_{30 \mathrm{~min}}\right.$ and $\left.\mathrm{O}_{60 \mathrm{~min}}\right)$. Figure 18 shows the results for seven different days of measurements and sampling frequencies. We observe that all dust opacities retrieved for each different sampling frequency is consistent with ODS error bars, showing therefore the reliability of the retrieval procedure for dust opacity for those sampling frequencies. In addition, for these different days we tested the sensitivity of ODS retrievals to the time data acquisition. In particular, we retrieved the dust optical depth using the 60minute sampling for the days analyzed in figure 18, and where the first observation of the day was set at 07:00 UTC (the subsequent observations are therefore performed at 08:00 UTC, 09:00 UTC...). Subsequently, a similar analysis is carried out but where the first observation of the 60-minute sampling is set at 08:15 UTC (then the observations are taken at 08:15 UTC, 09:15 UTC...), then set to 08:30 UTC and finally to 08:45 UTC. Results demonstrated that time data acquisition is of little impact on ODS retrievals, since similar results were found and consistent with ODS error bars.

\section{Clouds}

\subsection{Cloud properties retrieval procedure}

Clouds are detected by looking at the evolution of CI at twilight, since as mentioned above the presence of high clouds produces a minimum in the time variation of CI whose shape depends on the altitude and opacity of the cloud. In this work the twilight is defined as the time period for which SZA takes values between $86^{\circ}$ and $96^{\circ}$ (about 30-40 minutes duration). As for the dust opacity retrieval procedure, optical depth and altitude of clouds are derived from simulations of ODS signals. Since the simulations are undertaken at twilight and the plane parallel geometry approximation breaks down for high SZA, a radiative transfer model in spherical geometry is required. Our goal here is to specify the number of free parameters necessary to 
simulate the time variation of CI. A representative model of cloud for cirrus-type clouds is a thin layer at a given altitude with a spatial distribution of cloud particle density characterized by a Gaussian height profile, which is scaled to produce the required cloud optical depth. The scattering properties of cloud particles are described by the single scattering albedo and phase function. While the single scattering albedo of cloud particles at the ODS wavelengths is set to unity, the calculation of phase function represents a major challenge. Indeed, the shape of phase function depends, among other parameters, on the size and shape of particles. In particular, martian clouds are highly variable in particle size. Clancy et al., (2003) identified two different types of ice clouds from measurements of visible/IR opacity ratios. Ice clouds type 1 having particles sizes ranging between $1 \mu \mathrm{m}$ and $2 \mu \mathrm{m}$ and type 2 with sizes between $3 \mu \mathrm{m}$ and $4 \mu \mathrm{m}$. Subsequently, Montmessin et al., (2006) identified a third type of clouds exhibiting sizes between $0.1 \mu \mathrm{m}$ and $0.2 \mu \mathrm{m}$ from measurements carried out by the SPICAM instrument. Despite that these different ranges of sizes lead to different phase functions, a unique capacity of ODS colour index simulations is that the shape of the phase function has a little effect on the retrievals. Indeed, Toledo et al. (2016) showed that due to the fact that the CI is a relative measurement, the CI simulations are practically insensitive to the shape of the phase function, being the altitude and optical depth the major parameters in the simulations. Therefore, the total number of free parameters necessary to simulate clouds at twilight is 7: the cloud optical depth in the blue and red wavelength ranges $\left(\mathrm{COD}_{\text {blue }}, \mathrm{COD}_{\text {red }}\right)$, the cloud altitude $(\mathrm{h})$, the dust opacity in the blue and red wavelength ranges $\left(\tau_{\text {blue }}, \tau_{\text {red }}\right)$ and the effective radius and effective width $\left(\mathrm{r}_{\text {eff }}, v_{\text {eff }}\right)$ of the dust size distribution. However, the spectral dependence of COD is very small at the ODS wavelength ranges. In addition, since daytime ODS measurements constrain $\tau_{\text {blue }}, \tau_{\text {red }}, r_{\text {eff }}$ and $v_{\text {eff, }}$, the only free parameters in our model for cloud properties retrieval procedure are the opacity and altitude of the cloud.

Figure 19 shows the evolution of different CI signals simulated by using a threedimensional Monte-Carlo radiative transfer model (Trân, 2005) for different cloud altitudes and CODs, where the dust optical depth is fixed to 0.4. The dashed circles indicate the minimum in CI produced by the presence of clouds. Note that the shape of the CI signal strongly depends on cloud altitude and COD. Therefore, once a high cloud is detected by ODS, its altitude and optical depth can be estimated by searching the optimal values of these parameters that provides the best fit between simulated CI and ODS measured CI.

\subsection{Sampling strategy}

A major advantage of ODS instrument is its participation in several terrestrial campaigns through which the cloud properties retrieval procedure has been tested and evaluated. A good performance of ODS prototype on cirrus clouds detection and characterization was archived in Ouagadougou (Africa) during 2004-2005 (Toledo et al., 2016) and in Bauru (Brasil) from 2012 to 2013 (Toledo et al., 2015). Despite that in Earth the presence of high cloud produces a peak CI reddening instead of a minimum CI bluing, the principle of measurement is the same. As was done for the dust opacity retrieval procedure, we must evaluate the reliability of the retrieval procedure for different sampling frequencies. To this end, we analyzed different high clouds that were detected by ODS in Africa for five different days of measurements. Firstly, the COD and altitude for those clouds were retrieved using the original CI signals whose measurements were taken at 10 -second interval. Subsequently, the COD and altitude for these cases were retrieved but using only the observations of 
ODS signals that were taken at 1-minute interval, then at 2-minute interval and finally at 3-minute interval. We find that to be consistent with the retrievals undertaken using the original CI signals, ODS measurements must be taken at least at 1-minute interval. Figure 20 shows, as an example, the COD and cloud altitude retrieved by ODS using the original CI signals (black error bars) and those retrieved using the 1-minute, 2minute and 3-minute sampling frequencies. We observe that while the COD retrieved for the different sampling frequencies are consistent with ODS error bars, the 1minute sampling frequency is the only one that provides a cloud altitude within the error bars. Therefore, dual wavelength measurements must be obtained at least at 1min interval during the hour encompassing twilight.

\section{Conclusions}

In this paper we have demonstrated the capabilities of ODS (Optical Depth Sensor) to retrieve the daily average dust optical depth and the optical depth and altitude of clouds in the martian atmosphere. The procedure used to retrieve the dust optical depth is based on the modeling of the changes observed between the scattered flux at zenith and the sum of direct flux and scattered flux. By using radiative transfer simulations, the signals that should be observed by the instrument are simulated as a function of 4 free parameters: the dust optical depth in the blue and red wavelength ranges $\left(\tau_{\text {blue }}, \tau_{\text {red }}\right)$ and the effective radius and effective width ( $\left.\mathrm{r}_{\text {eff }}, v_{\text {eff }}\right)$ of the dust size distribution. The selection of these free parameters is based on the results obtained in different sensitivity analysis carried out on the ODS signals.

The simulations of ODS color index (CI) also demonstrate its capability to retrieve the opacity and altitude of clouds during sunrise and sunset. The principle of measurement is based on the minimum in the time variation of CI produced by the presence of clouds at SZAs between $86^{\circ}$ and $96^{\circ}$. As for dust opacity, the cloud parameters are retrieved using radiative transfer simulations but in this case the simulations are carried for the $\mathrm{CI}$ and at twilight. As demonstrated in different terrestrial campaigns, this procedure allows the detection of extremely thin clouds (Toledo et al., 2016) and at time periods for which cloud properties remain almost constant. However, it should be noted that ODS is designed rather for the characterization of ice aerosol layers partially homogeneous in space (at least within ODS field of view). This is to say, some limitations may be found in the characterization of clouds covering only a small part of ODS field of view or clouds with large spatial in-homogeneities. This assumption of spatial homogeneity (within ODS field of view) might not be a problem for the characterization of water ice aerosols, given the low-latitude aphelion season cloud belt (Clancy et al. 1996; Smith et al. 2013). However, we are realistic about the limitations of these measurements for the characterization of $\mathrm{CO}_{2}$ clouds for which the cloud cover can range between 20 and $500 \mathrm{~km}$ (Montmessin et al., 2007). In such cases, cloud opacity retrievals are weighted by the fraction of ODS field of view covered by the cloud.

In addition, the different retrieval procedures were tested against different sampling strategies. We found that dust opacities retrieved using single measurements at 60 minute interval are consistent with the ODS error bars. Results also demonstrate the capability of measurements at 30-minute interval to retrieve the tilting and orientation of the instrument. For the estimation of cloud properties, results indicate that ODS measurements should be taken at least at 1-minute interval in both channels. The daily sampling strategy proposed here consists in high frequency measurements at time intervals of at least 1-minute at twilight, and single measurements at time intervals of at least 60-minute during the day. 


\section{Acknowledgement}

This work was supported by the Centre National d'Études Spatiales (CNES) and the region of Champagne-Ardenne. 


\section{References}

Clancy, R. T., Grossman, A. W., Wolff, M. J., James, P. B., Rudy, D. J., Billawala, Y. N., Sandor, B. J., Lee, S. W. and Muhleman, D. O.: Water vapor saturation at low latitudes around aphelion: A key to Mars climate?. Icarus. 122, 36-62 (1996).

Clancy, R. T., Wolff, M. J. and Christensen, P. R.: Mars aerosol studies with the MGS TES emission phase function observations: Optical depths, particle sizes, and ice cloud types versus latitude and solar longitude. J. Geophys. Res. (2003). doi:10.1029/2003JE002058.

Colburn, D. S., Pollack, J. B. and Haberle, R. M.: Diurnal variations in optical depth at Mars. Icarus. (1989). doi:10.1016/0019-1035(89)90114-0.

Gierasch, P. J. and Goody, R. M.: The Effect of Dust on the Temperature of the Martian Atmosphere. J. Atmos. Sci. 29, 400-2 (1972).

Hansen, J. E. and Travis, L. D.: Light scattering in planetary atmospheres. Space Sci. Rev. (1974). doi:10.1007/BF00168069.

Korablev, O. I., Krasnopolsky, V. A. and Rodin, A. V.: Vertical structure of Martian dust measured by solar infrared occultations from the Phobos spacecraft. Icarus. 102, 76-87 (1993).

Lemmon, M. T., Wolff, M. J., Smith, M. D., Clancy, R. T., Banfield, D., Landis, G. A., Ghosh, A., Smith, P. H., Spanovich, N., Whitney, B., Whelley, P., Greeley, R., Thompson, S., Bell, J. F. and Squyres, S. W.: Atmospheric imaging results from the Mars exploration rovers: Spirit and Opportunity. Science. 306, 1753-6 (2004).

Lemmon, M. T., Wolff, M. J., Bell, J. F., Smith, M. D., Cantor, B. A. and Smith, P. H.: Dust aerosol, clouds, and the atmospheric optical depth record over 5 Mars years of the Mars Exploration Rover mission. Icarus. 251, 96-111 (2015).

Madeleine, J.-B., Forget, F., Millour, E., Montabone, L. and Wolff, M. J.: Revisiting the radiative impact of dust on Mars using the LMD Global Climate Model. J. Geophys. Res. (2011). doi:10.1029/2011JE003855.

Maria, J.-L., Tran, T. T., Pommereau, J.-P., Rannou, P., Malique, C., Correia, J. J. and Porteneuve, J.: Technical aspect of the optical depth sensor. Adv. Sp. Res. (2006). doi:10.1016/j.asr.2005.07.079.

Markiewicz, W. J., Sablotny, R. M., Keller, H. U., Thomas, N., Titov, D. and Smith, P. H.: Optical properties of the Martian aerosols as derived from Imager for Mars Pathfinder midday sky brightness data. J. Geophys. Res. (1999). doi:10.1029/1998JE900033.

Medvedev A. S., Kuroda T. and Hartogh P.: Influence of dust on the dynamics of the martian atmosphere above the first scale height. Aeolian Research. 3, 145-156 (2011). 
Mishchenko, M. I., Travis, L. D., Kahn, R. A. and West, R. A.: Modeling phase functions for dust-like tropospheric aerosols using a shape mixture of randomly oriented polydisperse spheroids. J. Geophys. Res. (1997). doi:10.1029/97JD01124.

Montmessin, F., Rannou, P. and Cabane, M.: New insights into Martian dust distribution and water-ice cloud microphysics. J. Geophys. Res. (2002).

doi:10.1029/2001JE001520.

Montmessin, F., Quémerais, E., Bertaux, J. L., Korablev, O., Rannou, P. and Lebonnois, S.: Stellar occultations at UV wavelengths by the SPICAM instrument: Retrieval and analysis of Martian haze profiles. J. Geophys. Res. (2006). doi:10.1029/2005JE002662.

Montmessin, F., Gondet, B., Bibring, J.-P., Langevin, Y., Drossart, P., Forget, F. and Fouchet, T.: Hyperspectral imaging of convective $\mathrm{CO} 2$ ice clouds in the equatorial mesosphere of Mars. J. Geophys. Res. (2007). doi:10.1029/2007JE002944 (2007).

Pollack, J. B., Colburn, D. S., Flasar, F. M., Kahn, R., Carlston, C. E. and Pidek, D.: Properties and effects of dust particles suspended in the Martian atmosphere. J. Geophys. Res. 84, 2929- 2945 (1979).

Pollack, J. B. and Cuzzi, J. N.: Scattering by nonspherical particles of size comparable to wavelength - A new semi-empirical theory and its application to tropospheric aerosols. (1980). doi:10.1175/1520-0469(1980)037<0868:SBNPOS>2.0.CO;2.

Pollack, J. B., Ockert-Bell, M. E. and Shepard, M. K.: Viking Lander image analysis of Martian atmospheric dust. J. Geophys. Res. (1995). doi:10.1029/94JE02640.

Sarkissian, A., Pommereau, J. P. and Goutail, F.: Identification of polar stratospheric clouds from the ground by visible spectrometry. Geophys. Res. Lett. (1991). doi:10.1029/91GL00769.

Smith, M. D., Wolff, M. J., Lemmon, M. T., Spanovich, N., Banfield, D., Budney, C. J., Clancy, R. T., Ghosh, A., Landis, G. A., Smith, P., Whitney, B., Christensen, P. R. and Squyres, S. W.: First Atmospheric Science Results from the Mars Exploration Rovers Mini-TES. Science. 306(5702), 1750-3 (2004).

Smith, M. D.: THEMIS observations of Mars aerosol optical depth from 2002-2008. Icarus. (2009). doi:10.1016/j.icarus.2009.03.027.

Smith, M.D., Wolff, M.J., Clancy, R.T., Kleinbohl, A., Murchie, S.L.: Vertical Distribution of Dust and Water Ice Aerosols from CRISM Limb-Geometry Observations. J. Geophys. Res. 118 (E2), 321-334 (2013).

Smith, P. H. and Lemmon, M.: Opacity of the Martian atmosphere measured by the Imager for Mars Pathfinder. J. Geophys. Res. (1999). doi:10.1029/1998JE900017.

Stamnes, K., Tsay, S. C., Wiscombe, W. and Jayaweera, K.: Numerically stable algorithm for discrete-ordinate-method radiative transfer in multiple scattering and emitting layered media. Appl. Opt. (1988). doi:10.1364/AO.27.002502. 
Toledo, D.: Preparation and validation of the cloud and dust opacity sensor ODS for ExoMars 2018 mission. PhD thesis in Astrophysics, Univ. Reims ChampagneArdenne (2015).

Toledo, D., Rannou, P., Pommereau, J-P., Sarkissian, A., and Foujols, T.: Measurement of aerosol optical depth and sub-visual cloud detection using the optical depth sensor (ODS). Atmos. Meas. Tech. (2016). doi/10.5194/amt-9-455-2016.

Tomasko, M. G., Doose, L. R., Lemmon, M., Smith, P. H. and Wegryn, E.: Properties of dust in the Martian atmosphere from the Imager on Mars Pathfinder. J. Geophys. Res. (1999). doi:10.1029/1998JE900016.

Tran, T. T., Pommereau, J.-P., Rannou, P. and Maria, J.-L.: Scientific aspects of the optical depth sensor. Adv. Sp. Res. (2005). doi:10.1016/j.asr.2005.08.021.

Trân, T.-T.: Optical Depth Sensor for Measurement of Dust and Clouds in the Atmosphere of Mars: Radiative Transfer Simulations and Validation on Earth. Thèse Dr. (Astrophysique). Univ. Versailles St-Quentin en Yvelines. (2005).

Wolff, M. J., Smith, M. D., Clancy, R. T., Spanovich, N., Whitney, B. A., Lemmon, M. T., Bandfield, J. L., Banfield, D., Ghosh, A., Landis, G., Christensen, P. R., Bell, J. F. and Squyres, S. W.: Constraints on dust aerosols from the Mars Exploration Rovers using MGS overflights and Mini-TES. J. Geophys. Res. (2006). doi:10.1029/2006JE002786.

Wolff, M. J., Smith, M. D., Clancy, R. T., Arvidson, R., Kahre, M., Seelos, F., Murchie, S. and Savijärvi, H.: Wavelength dependence of dust aerosol single scattering albedo as observed by the Compact Reconnaissance Imaging Spectrometer. J. Geophys. Res. (2009). doi:10.1029/2009JE003350. 


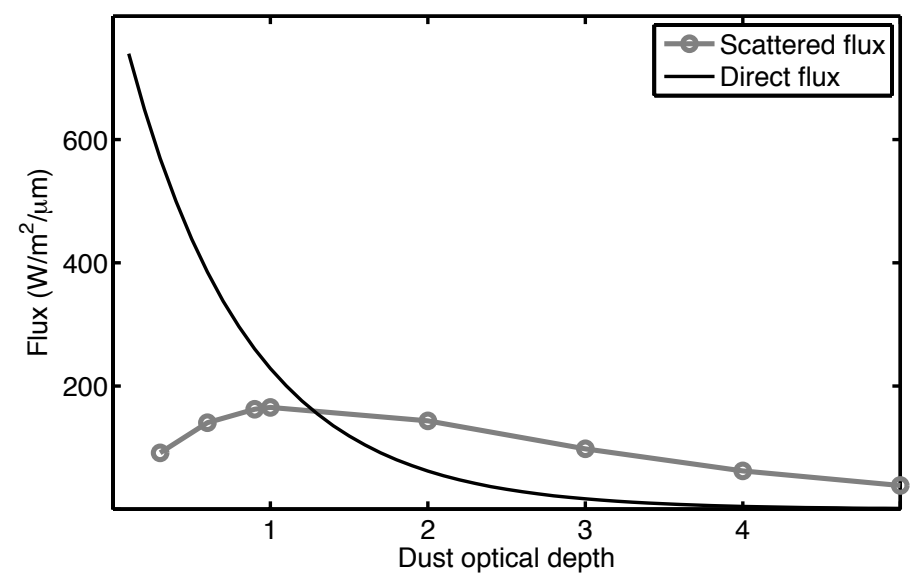

Figure 1. Simulated solar flux on Mars surface as a function of dust opacity. 


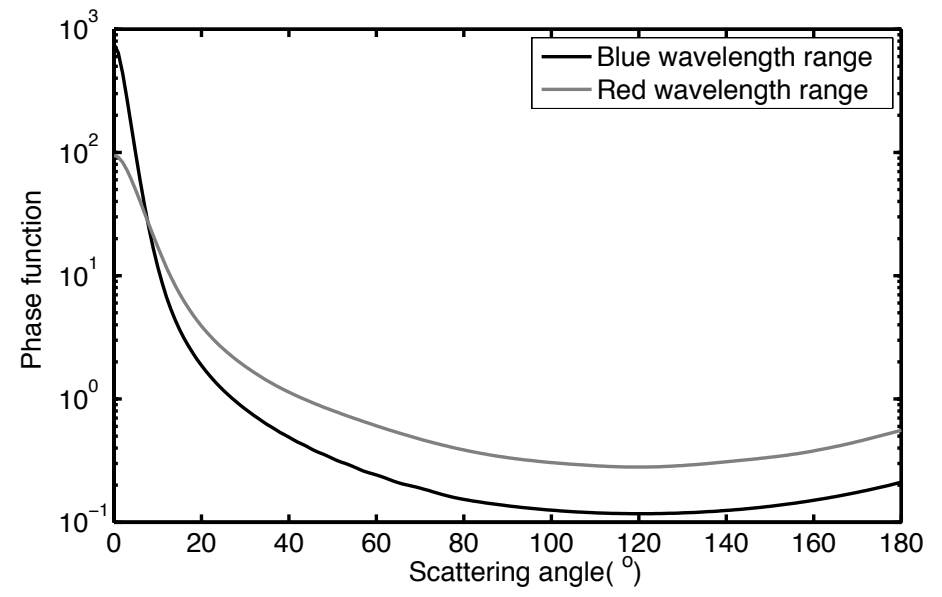

Figure 2. Phase functions of martian dust in the blue and red wavelength ranges calculated by the empirical formulation proposed by Pollack and Cuzzi (1980). 

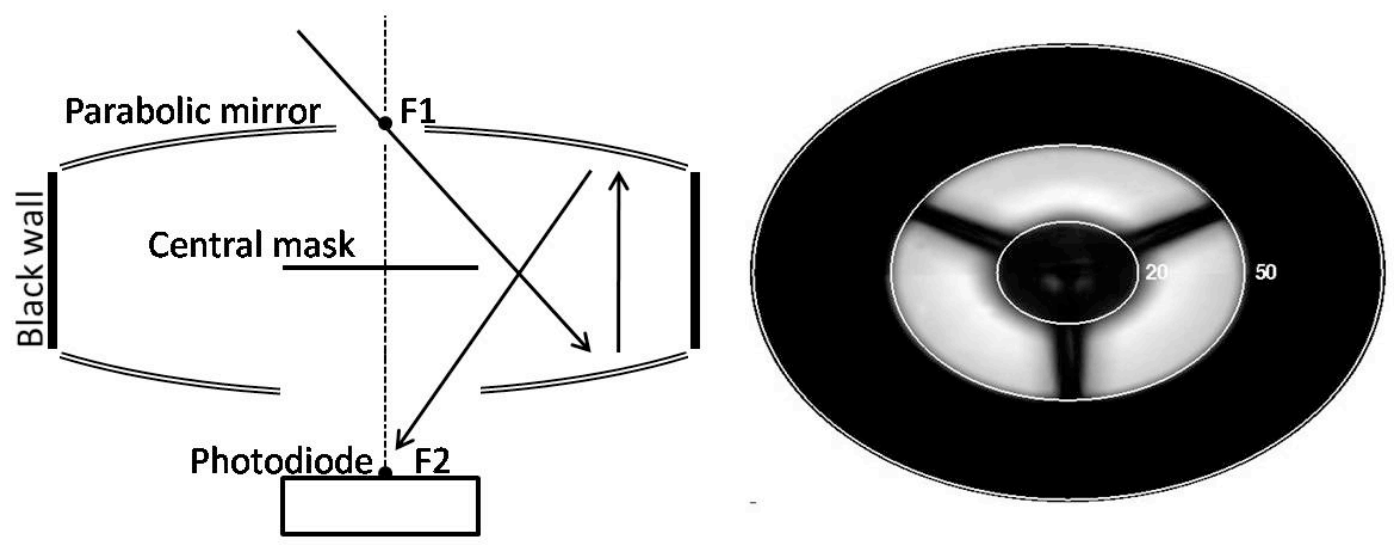

Figure 3. ODS optical head (on the left) made by two parabolic mirrors surrounded by black walls to avoid unwanted reflexions, a central mask supported by 3 small legs and a photodiode. Rays entering the entrance hole at the focal point F1 (black arrows), are reflected by the parabolic mirrors and focused on the photodiode focal point F2. Right: measured ODS field of view. White and black areas correspond to $100 \%$ and 0 $\%$ of transmission, respectively. Most light is collected between $25^{\circ}$ and $50^{\circ}$. 

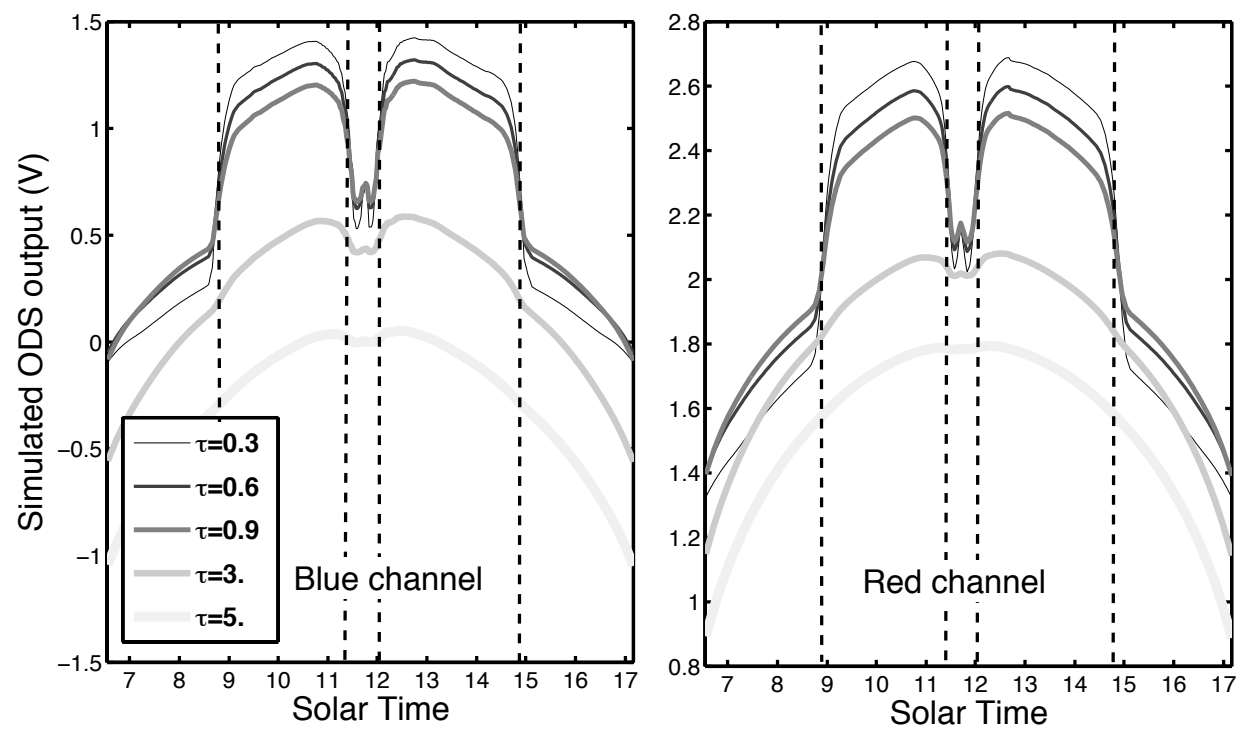

Figure 4. (a) Simulated daytime ODS output voltage in the blue channel for different dust opacities: $0.3,0.6,0.9,3$ and 5. (b) is the same as (a) but for the red channel. The simulations are undertaken for fixed values of $\mathrm{r}_{\text {eff }}=1.6 \mu \mathrm{m}, v_{\text {eff }}=0.2, \mathrm{SLP}_{\text {blue }}=115$, $\operatorname{SLP}_{\text {red }}=120, \theta_{\text {min }}^{\text {blue }}=138^{\circ}$ and $\theta_{\text {min }}^{\text {red }}=150^{\circ}$. The bands delimited by black dashed lines represent the time intervals for which the sun is within the FOV of ODS. 

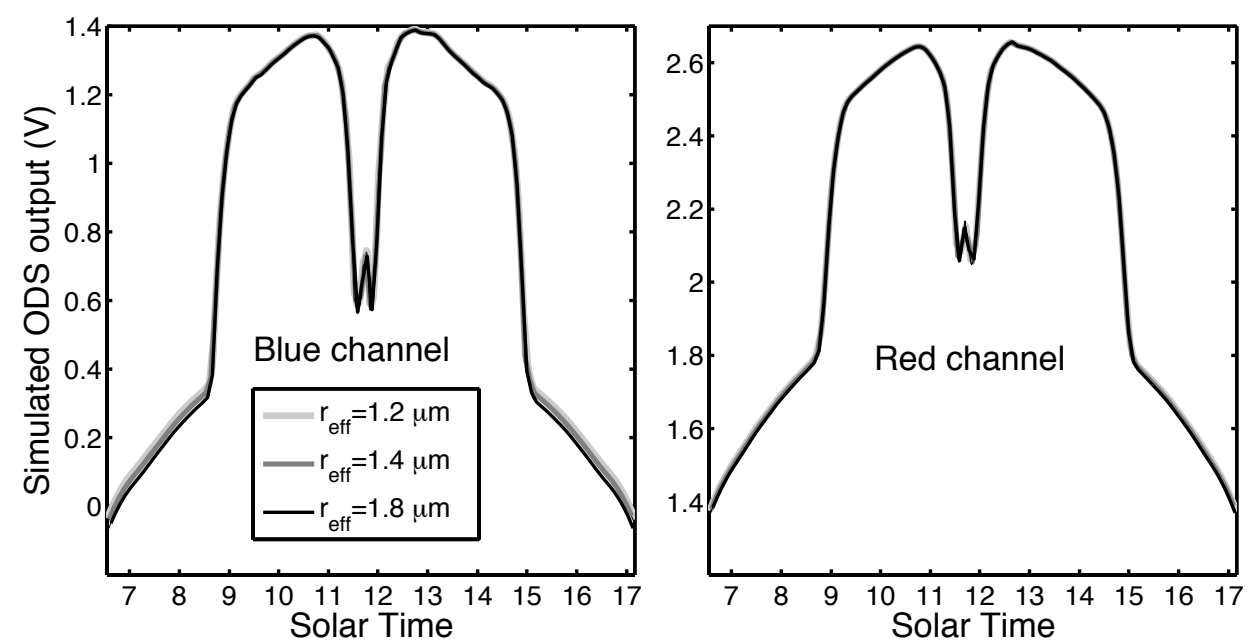

Figure 5. (a) Simulated daytime blue ODS output voltage for a total opacity $\tau=0.4$ and for different values of $\mathrm{r}_{\text {eff: }}: 1 . \mu \mathrm{m}, 1.2 \mu \mathrm{m}, 1.4 \mu \mathrm{m}$ and $1.8 \mu \mathrm{m}$. (b) the same as (a) but for the red channel. The simulations are undertaken for same values of $v_{\text {eff }}, S_{\text {SPlue, }}$ $\mathrm{SLP}_{\text {red }}, \theta^{\text {blue }}{ }_{\min }$ and $\theta_{\text {min }}^{\text {red }}$ as used in Figure 4. 


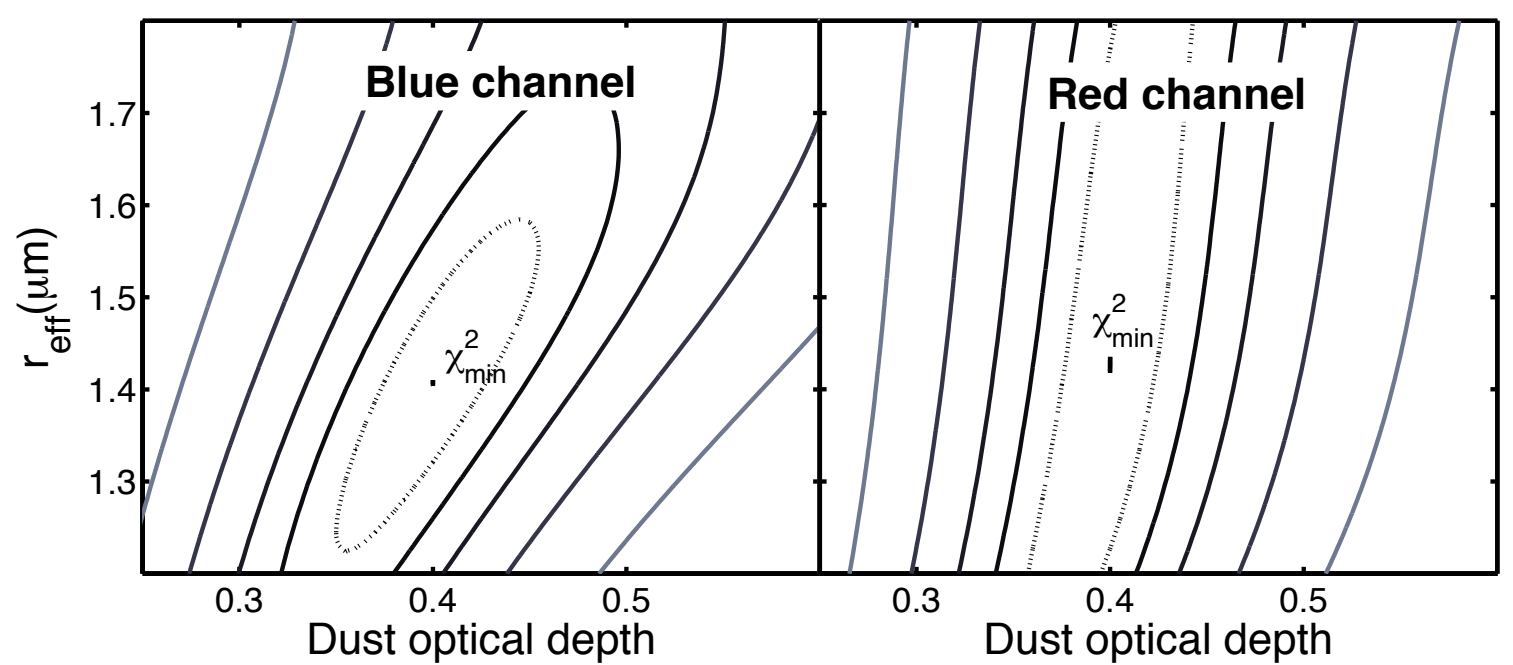

Figure 6. Contours of $\chi^{2}$ in the $\tau-r_{\text {eff }}$ space, and where $\mathrm{O}_{\text {Mars }}$ signal in the blue and red channels was computed for $\tau=0.4$ and $r_{\text {eff }}=1.4 \mu \mathrm{m}$. The value of $\chi^{2}$ for the different contour lines is given by $\chi^{2}=2^{k} \cdot \chi^{2}{ }_{\min }(k=1,2 \ldots)$. The dash-dot lines correspond to the case $\mathrm{k}=1$. 

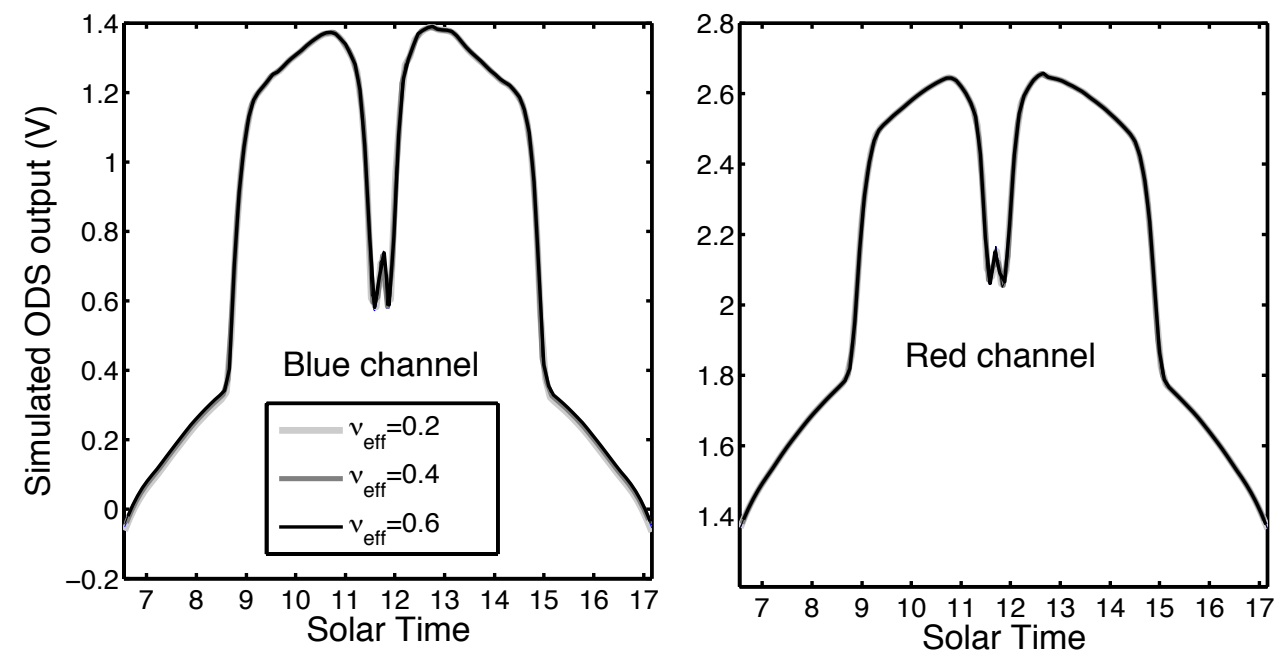

Figure 7. (a) Simulated daytime blue ODS output voltage for a total opacity $\tau=0.4$ and for different values of $v_{\text {eff: }} 0.2,0.4$ and 0.6. (b) is the same as (a) but for the red channel. The simulations are undertaken for same values of $r_{\text {eff }}, \mathrm{SLP}_{\text {blue }}, \mathrm{SLP}_{\text {red, }}$ $\theta_{\min }^{\text {blue }}$ and $\theta_{\text {min }}^{\text {red }}$ as used in Figure 4. 


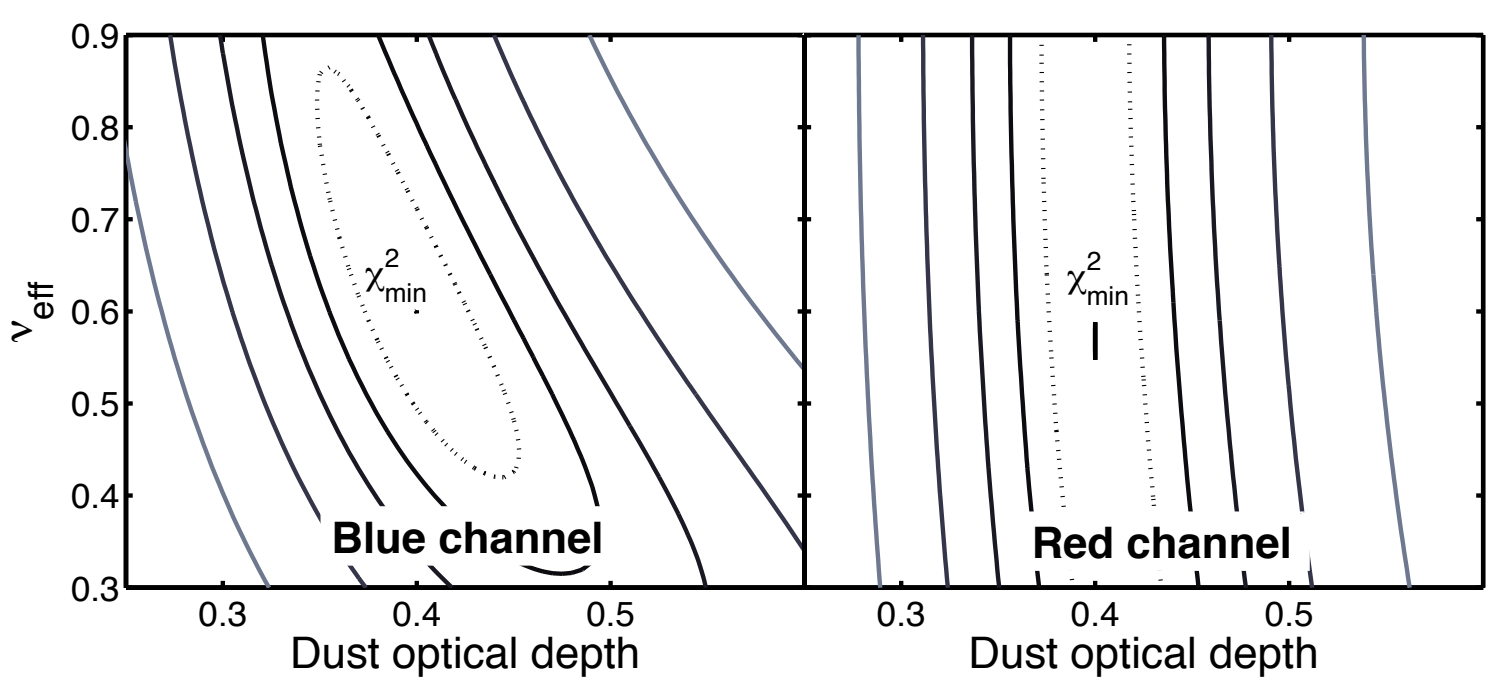

Figure 8. Contours of $\chi^{2}$ in the $\tau-v_{\text {eff }}$ space, and where $\mathrm{O}_{\text {Mars }}$ signal in the blue and red channels was computed for $\tau=0.4$ and $v_{\text {eff }}=0.6$. The value of $\chi^{2}$ for the different contour lines is given by $\chi^{2}=2^{k} \cdot \chi^{2}{ }_{\min }(k=1,2 \ldots)$. The dash-dot lines correspond to the case $\mathrm{k}=1$. 

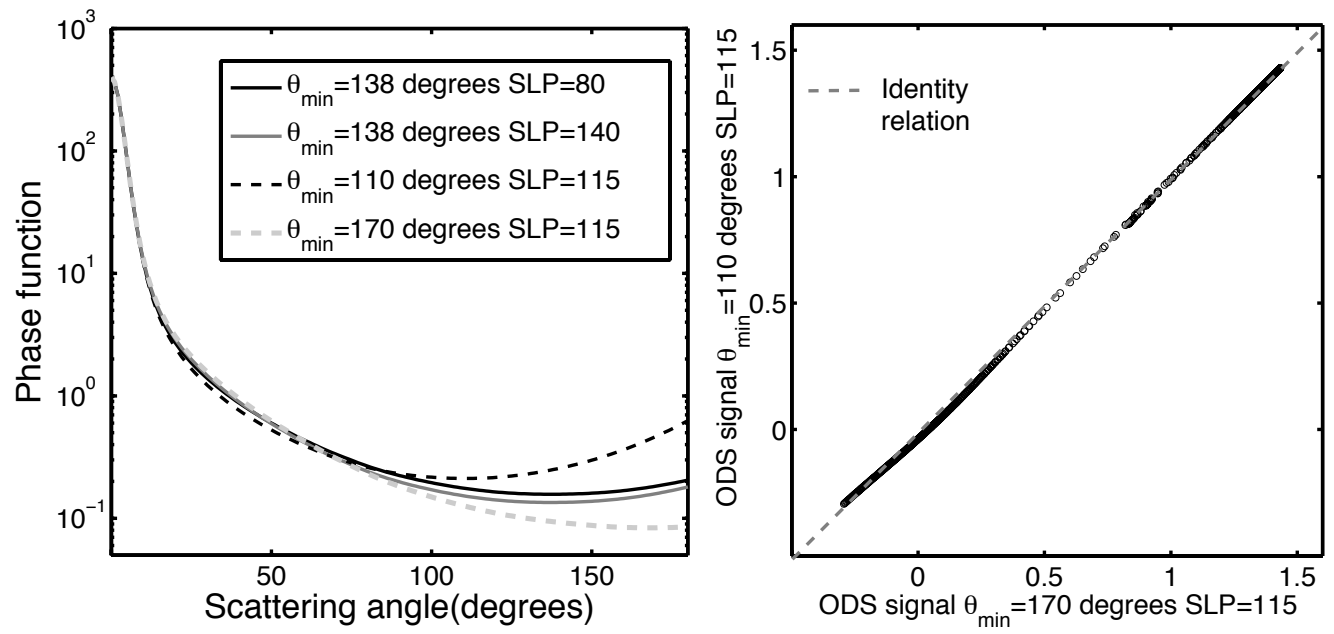

Figure 9. Left) Phase functions in the blue wavelength range calculated for different values of $\theta_{\min }$ and SLP. Right) Correlation between ODS signals simulated for $\theta_{\min }=170^{\circ}$ and $\mathrm{SLP}=115$, and $\theta_{\min }=110^{\circ}$ and $\mathrm{SLP}=115$. The grey dashed line represents the identity relation. 

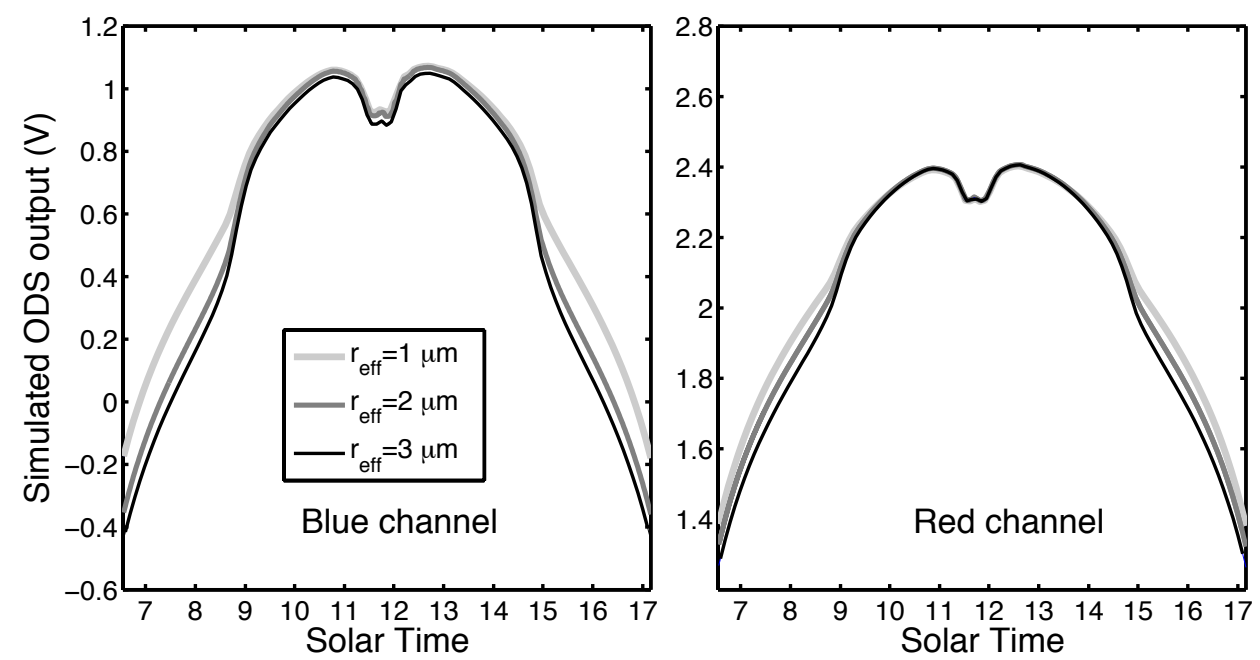

Figure 10. (a) Simulated daytime ODS output voltage for the blue channel for different values of $r_{\text {eff: }} 1 \mu \mathrm{m}, 2 \mu \mathrm{m}$ and $3 \mu \mathrm{m}$. (b) is the same as (a) but for the red channel. The simulations are undertaken for $v_{\text {eff }}=0.4, \tau=2$ and $\operatorname{SLP}_{\text {blue }}, \operatorname{SLP}_{\text {red }}, \theta_{\text {min }}^{\text {blue }}$ and $\theta_{\min }^{\text {red }}$ as used in Figure 4. 


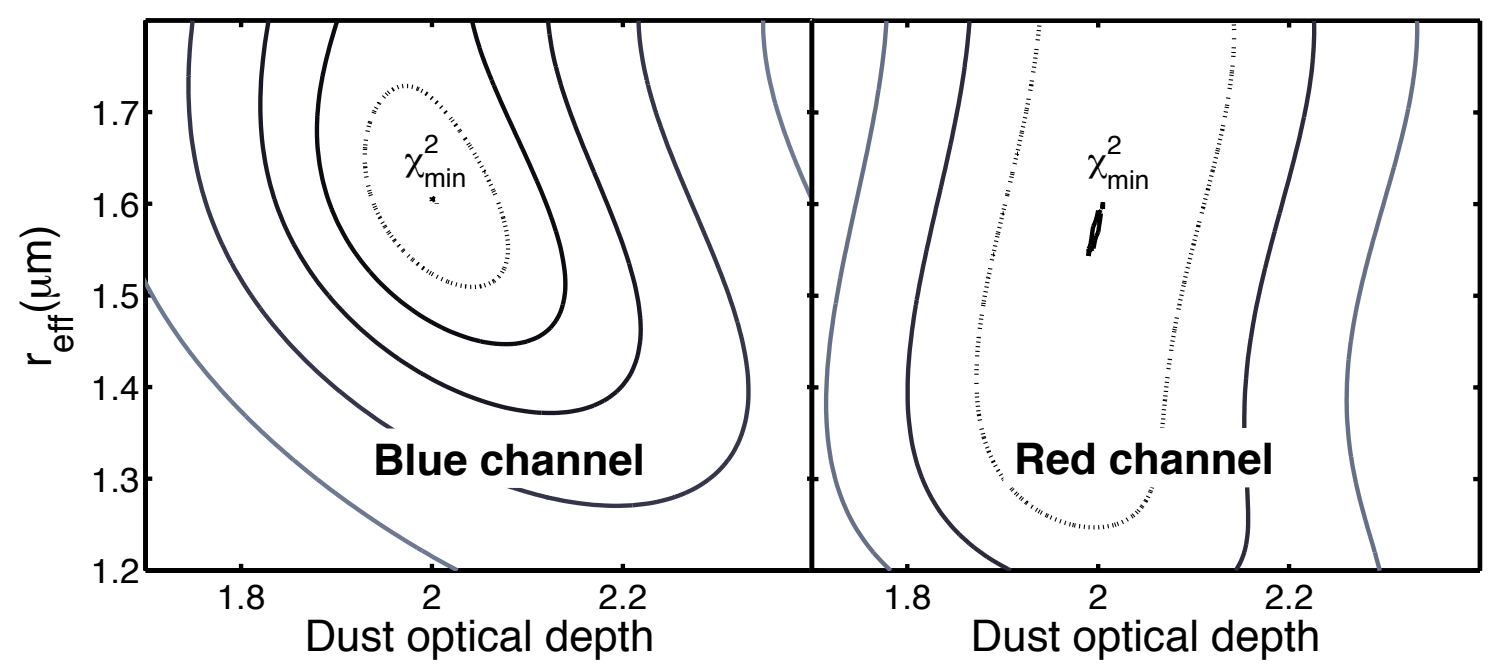

Figure 11. Same as Figure 6 but for $\tau=2$. and $r_{\text {eff }}=1.6 \mu \mathrm{m}$. 

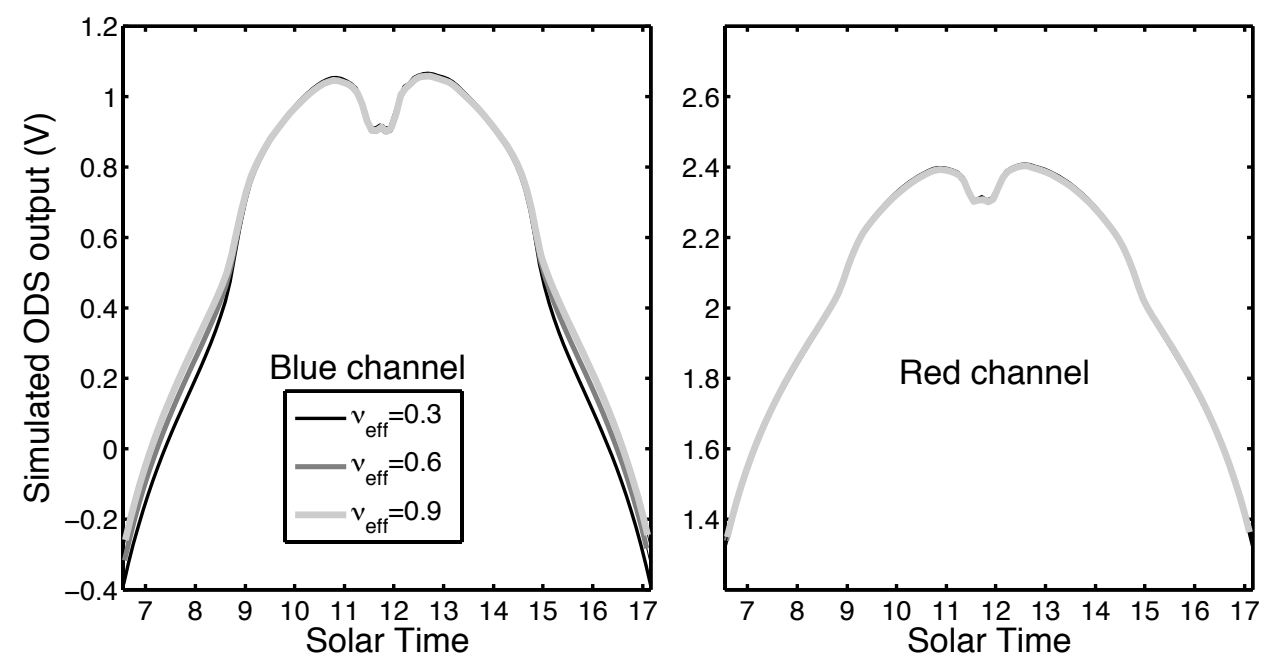

Figure 12. (a) Simulated daytime ODS output voltage for the blue channel for different values of $v_{\text {eff: }}: 0.3,0.6$ and 0.9. (b) is the same as (a) but for the red channel. The simulations are undertaken for $\mathrm{r}_{\text {eff }}=2 \mu \mathrm{m}, \tau=2$ and $\mathrm{SLP}_{\text {blue }}, \mathrm{SLP}_{\text {red }}, \theta_{\text {min }}^{\text {blue }}$ and $\theta_{\text {min }}^{\text {red }}$ as used in figure 4. 


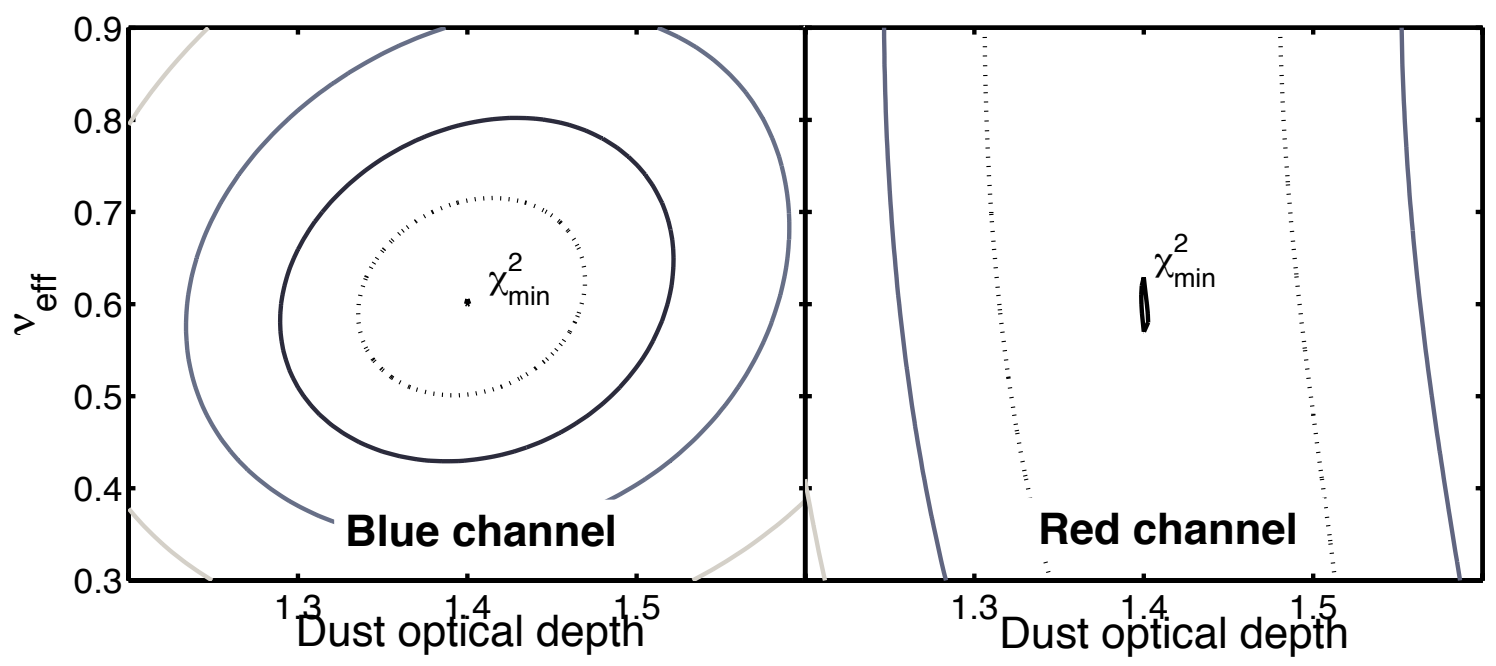

Figure 13. Same as Figure 8 but for $\tau=1.4$ and $v_{\text {eff }}=0.6$. 


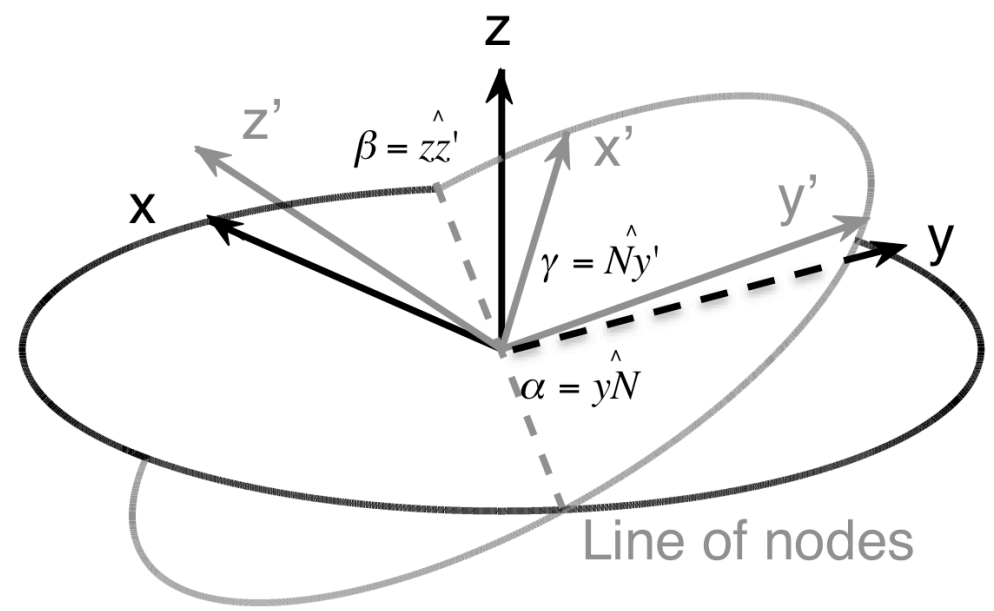

Figure 14. Euler angles of rotation transforming the system $\mathrm{L}\{\mathrm{x}, \mathrm{y}, \mathrm{z}\}$ into the new coordinate system $\mathrm{O}\left\{\mathrm{x}^{\prime}, \mathrm{y}^{\prime}, \mathrm{z}^{\prime}\right\}$. The grey dashed line represents the line of nodes. 

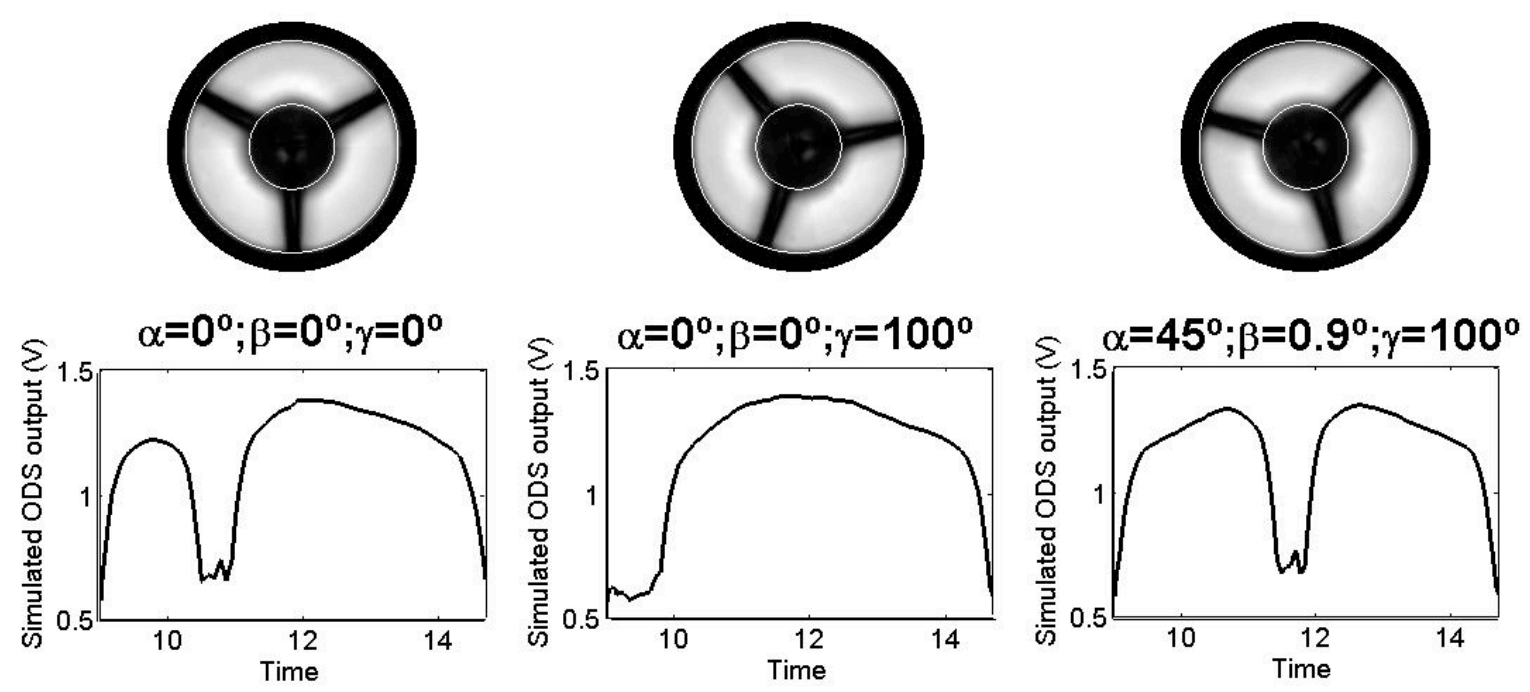

Figure 15. Simulated ODS signals for different angles of tilting and orientations of the instrument. 


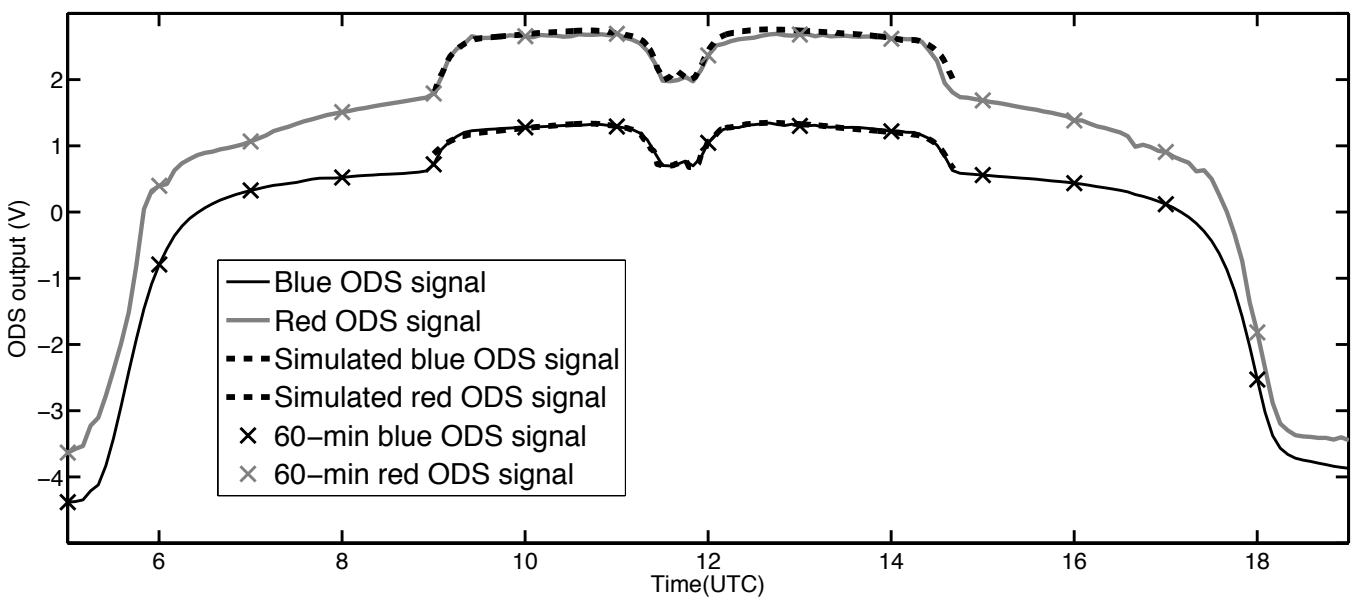

Figure 16. ODS measurements at Ouagadougou (Africa) on 23 December 2004 (black and grey lines) and best fit results of the angles of rotation (black dashed lines). The black and grey crosses represent ODS observations acquired at 60-minute interval. 


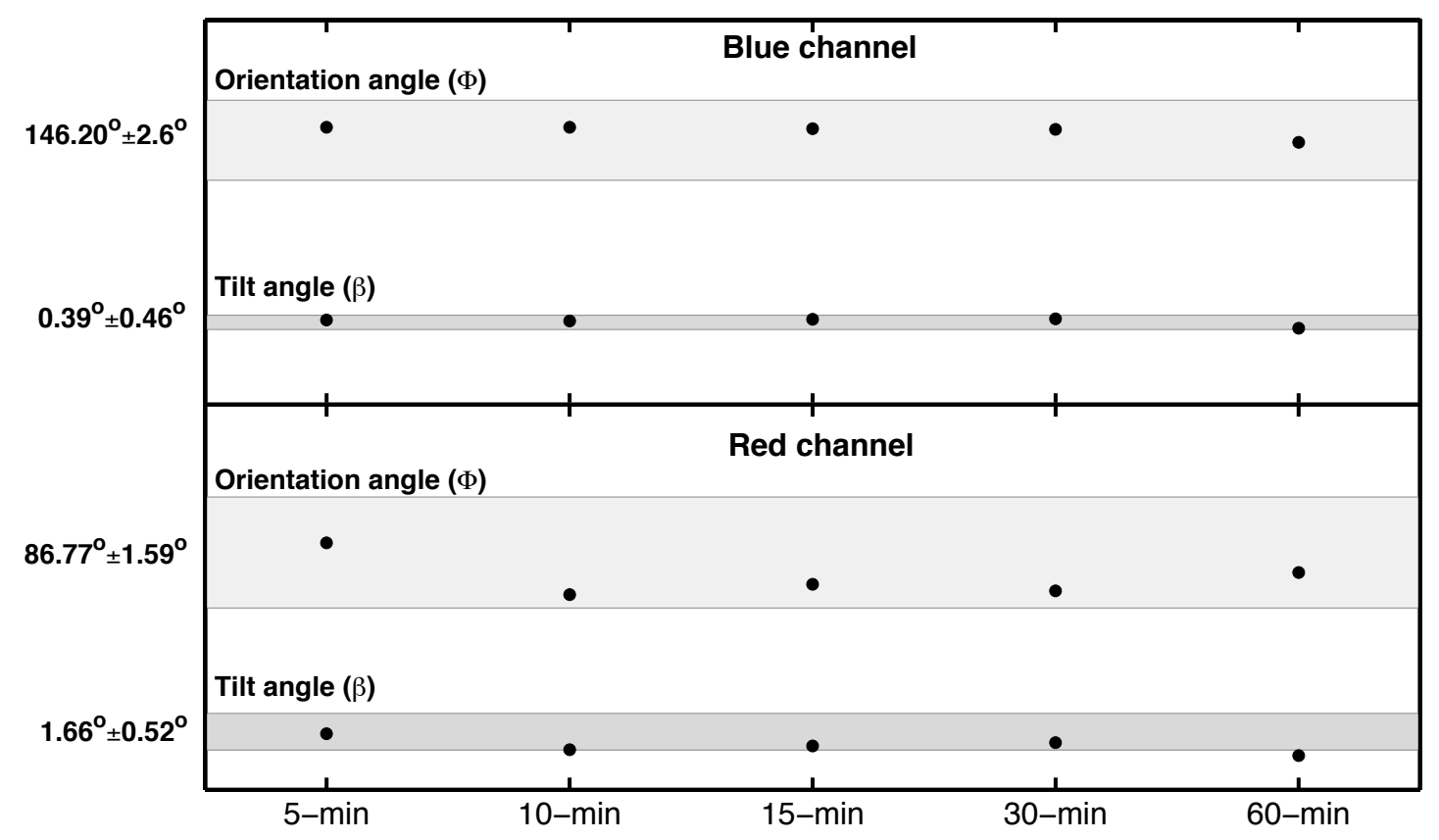

Figure 17. Euler angles and orientation angle $\Phi$ retrieved using the original ODS signals (grey bars) and those retrieved using only the observations that were taken at 5-minute interval, 10-minute interval, 15-minute interval, 30-minute interval and 60minute interval. 

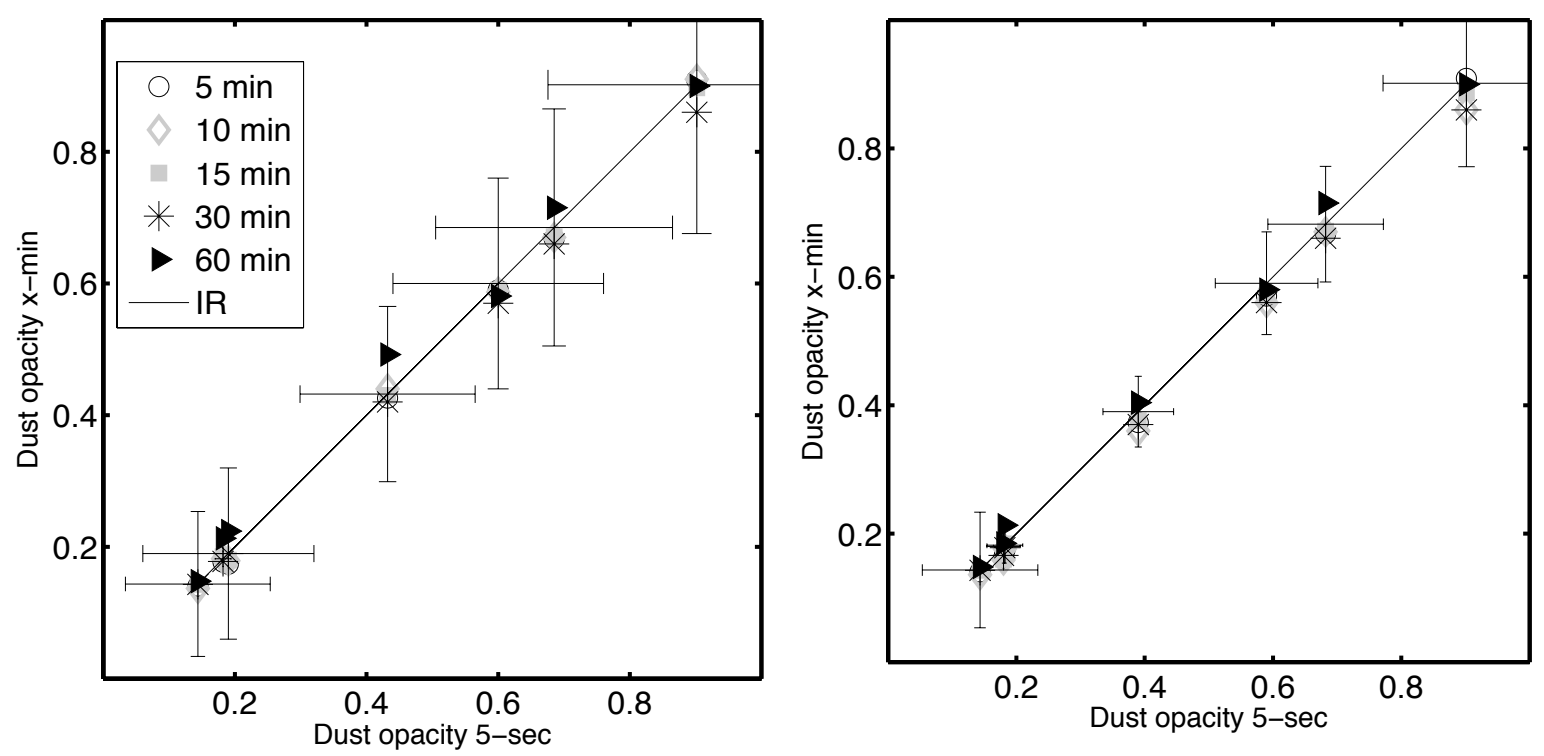

Figure 18. Correlation between the dust optical depths retrieved using all observations of ODS signals (black error bars) and those retrieved using only the observations that were taken at 5-minute interval, 10-minute interval, 15-minute interval, 30-minute interval and 60-minute interval. Black line (IR) represents the identity relation. 


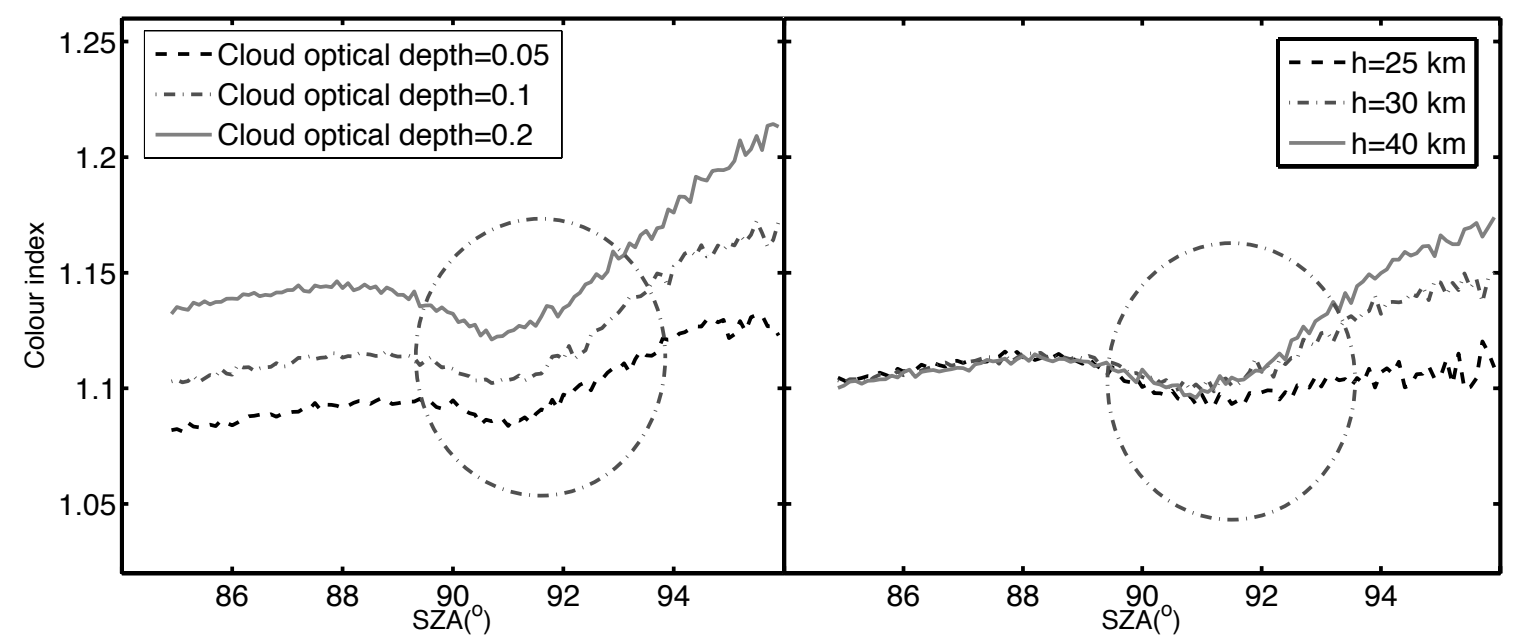

Figure 19. (a) Simulated colour index during twilight for cloud altitude $\mathrm{h}=35 \mathrm{~km}$ and cloud optical depth $\mathrm{COD}=0.05,0.1$ and 0.2. (b) same as (a) but for $\mathrm{COD}=0.1$ and $\mathrm{h}=25 \mathrm{~km}, 30 \mathrm{~km}$ and $40 \mathrm{~km}$. 

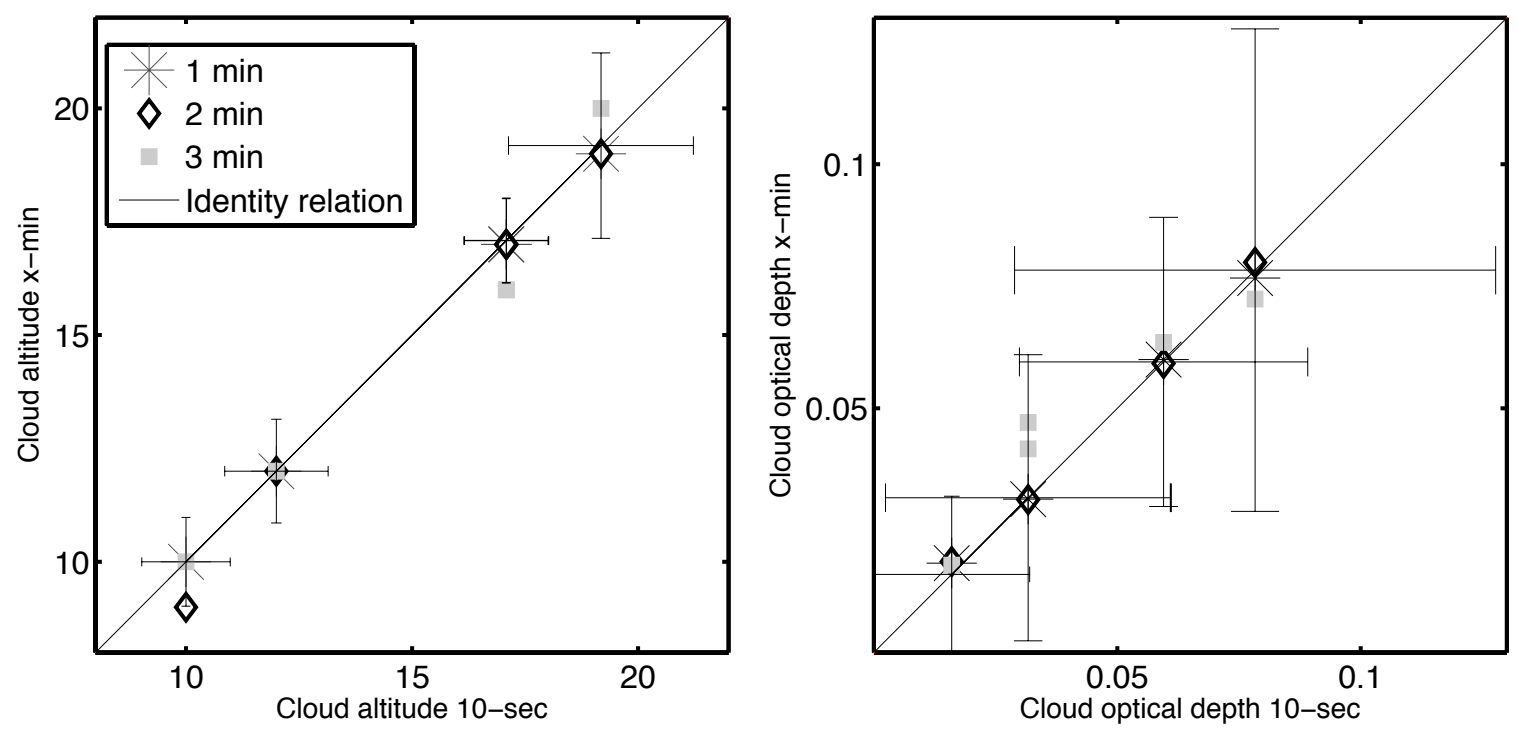

Figure 20. Correlation between the retrieved cloud altitudes and CODs using all observations of ODS signals (red error bars) and those retrieved using only the observations that were taken at 1-minute interval, 2-minute interval and 3-minute interval. 
Table 1. Single scattering albedo $(\omega)$ and asymmetry parameter $(\mathrm{g})$ of the phase function calculated for different $r_{\text {eff }}$ using the empirical method of Pollack and Cuzzi.

\begin{tabular}{clll}
\hline $\mathrm{r}_{\text {eff }}$ & $1.2 \mu \mathrm{m}$ & $1.4 \mu \mathrm{m}$ & $1.8 \mu \mathrm{m}$ \\
\hline$\omega_{\text {blue }}$ & 0.79 & 0.77 & 0.74 \\
$\omega_{\text {red }}$ & 0.95 & 0.94 & 0.94 \\
$\mathrm{~g}_{\text {blue }}$ & 0.75 & 0.76 & 0.79 \\
$\mathrm{~g}_{\text {red }}$ & 0.70 & 0.70 & 0.71 \\
\hline
\end{tabular}


Table 2. Single scattering albedo $(\omega)$ and asymmetry parameter $(\mathrm{g})$ of the phase function calculated for different $v_{\text {eff }}$ using the empirical method of Pollack and Cuzzi.

\begin{tabular}{cccc}
\hline$v_{\text {eff }}$ & 0.2 & 0.4 & 0.6 \\
\hline$\omega_{\text {blue }}$ & 0.75 & 0.77 & 0.78 \\
$\omega_{\text {red }}$ & 0.94 & 0.94 & 0.94 \\
$\mathrm{~g}_{\text {blue }}$ & 0.78 & 0.77 & 0.76 \\
$\mathrm{~g}_{\text {red }}$ & 0.71 & 0.71 & 0.71 \\
\hline
\end{tabular}


Table 3. Single scattering albedo $(\omega)$ and asymmetry parameter $(\mathrm{g})$ of the phase function calculated for different $r_{\text {eff }}$ using the empirical method of Pollack and Cuzzi.

\begin{tabular}{cccc}
\hline $\mathrm{r}_{\text {eff }}$ & $1 \mu \mathrm{m}$ & $2 \mu \mathrm{m}$ & $3 \mu \mathrm{m}$ \\
\hline$\omega_{\text {blue }}$ & 0.82 & 0.74 & 0.70 \\
$\omega_{\text {red }}$ & 0.96 & 0.93 & 0.90 \\
$\mathrm{~g}_{\text {blue }}$ & 0.75 & 0.80 & 0.84 \\
$\mathrm{~g}_{\text {red }}$ & 0.70 & 0.72 & 0.74 \\
\hline
\end{tabular}


Table 4. Single scattering albedo $(\omega)$ and asymmetry parameter $(\mathrm{g})$ of the phase function calculated for different $r_{\text {eff }}$ using the empirical method of Pollack and Cuzzi.

\begin{tabular}{cccc}
\hline$v_{\text {eff }}$ & 0.3 & 0.6 & 0.9 \\
\hline$\omega_{\text {blue }}$ & 0.73 & 0.75 & 0.77 \\
$\omega_{\text {red }}$ & 0.93 & 0.93 & 0.93 \\
$\mathrm{~g}_{\text {blue }}$ & 0.80 & 0.78 & 0.77 \\
$\mathrm{~g}_{\text {red }}$ & 0.72 & 0.72 & 0.72 \\
\hline
\end{tabular}


Table 5. Retrieved rotations: Euler angles $(\alpha, \beta, \gamma)$ and orientation angle $\Phi$.

$\begin{array}{ccc}\text { Angles } & \text { Blue channel } & \text { Red channel } \\ \alpha & 108.14^{\circ} \pm 2.18^{\circ} & 8.35^{\circ} \pm 0.82^{\circ} \\ \beta & 0.39^{\circ} \pm 0.46^{\circ} & 1.66^{\circ} \pm 0.52^{\circ} \\ \gamma & 38.05^{\circ} \pm 1.60^{\circ} & 78.41^{\circ} \pm 1.36^{\circ} \\ \Phi & 146.20^{\circ} \pm 2.6^{\circ} & 86.77^{\circ} \pm 1.59^{\circ}\end{array}$

